\title{
Experimental investigation of auroral generator regions with conjugate Cluster and FAST data
}

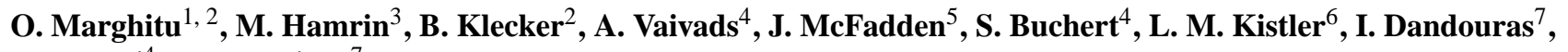 \\ M. André ${ }^{4}$, and H. Rème ${ }^{7}$ \\ ${ }^{1}$ Institute for Space Sciences, Bucharest, Romania \\ ${ }^{2}$ Max-Planck-Institut für extraterrestrische Physik, Garching, Germany \\ ${ }^{3}$ Department of Physics, Umeå University, Umeå, Sweden \\ ${ }^{4}$ Swedish Institute of Space Physics, Uppsala, Sweden \\ ${ }^{5}$ Space Sciences Lab., University of California at Berkeley, USA \\ ${ }^{6}$ Space Science Center, University of New Hampshire, Durham, USA \\ ${ }^{7}$ CESR-CNRS, Toulouse, France
}

Received: 19 April 2005 - Revised: 21 November 2005 - Accepted: 10 January 2006 - Published: 23 March 2006

\begin{abstract}
Here and in the companion paper, Hamrin et al. (2006), we present experimental evidence for the crossing of auroral generator regions, based on conjugate Cluster and FAST data. To our knowledge, this is the first investigation that concentrates on the evaluation of the power density, $\boldsymbol{E} \cdot \boldsymbol{J}$, in auroral generator regions, by using in-situ measurements. The Cluster data we discuss were collected within the Plasma Sheet Boundary Layer (PSBL), during a quiet magnetospheric interval, as judged from the geophysical indices, and several minutes before the onset of a small substorm, as indicated by the FAST data. Even at quiet times, the PSBL is an active location: electric fields are associated with plasma motion, caused by the dynamics of the plasma-sheet/lobe interface, while electrical currents are induced by pressure gradients. In the example we show, these ingredients do indeed sustain the conversion of mechanical energy into electromagnetic energy, as proved by the negative power density, $\boldsymbol{E} \cdot \boldsymbol{J}<0$. The plasma characteristics in the vicinity of the generator regions indicate a complicated 3-D wavy structure of the plasma sheet boundary. Consistent with this structure, we suggest that at least part of the generated electromagnetic energy is carried away by Alfvén waves, to be dissipated in the ionosphere, near the polar cap boundary. Such a scenario is supported by the FAST data, which show energetic electron precipitation conjugated with the generator regions crossed by Cluster. A careful examination of the conjunction timing contributes to the validation of the generator signatures.
\end{abstract}

Keywords. Magnetospheric physics (Auroral phenomena; Magnetosphere-ionosphere interactions; Magnetotail boundary layers)

Correspondence to: O. Marghitu

(marghitu@ venus.nipne.ro)

\section{Introduction}

Within an auroral generator region mechanical energy of the thermal and/or bulk plasma motion is converted into electromagnetic energy. This energy is transported as Poynting flux along the auroral magnetic field lines, and converted back into mechanical energy at lower altitudes. Several studies addressed the auroral generator by using analytical (e.g. Rostoker and Boström, 1976), semi-analytical (e.g. Lysak, 1985; Vogt et al., 1999), and numerical tools (e.g. Birn et al., 1996; Birn and Hesse, 1996). We should point out that the term "auroral generator" is associated in the literature both with the generation of electromagnetic energy and field-aligned current (FAC). In this study and in the companion paper, Hamrin et al. (2006), referred below as H06, we concentrate only on the generation of electromagnetic energy. The generation of FAC is beyond the scope of these papers.

To our knowledge the key generator feature, expressed in the relation $\boldsymbol{E} \cdot \boldsymbol{J}<0$, has not been investigated yet by experimental means. This can be understood by noting that both the current density and the electric field are difficult to measure in those magnetospheric domains where generator processes are expected. In order to derive the full current density vector, by computing the curl of the magnetic field, at least four satellites are needed. In addition, even for a strong aurora the energy flux into the ionosphere is consistent with quite small generator power densities, implying currents and electric fields close to the instrumental detection limit.

The examination of conjugate nightside data from the Cluster fleet (at an altitude of $\sim 18 R_{E}$ ) and FAST (at $\sim 0.6 R_{E}$ ) provides a good opportunity for the investigation of auroral generator regions. Because the Cluster mission consists of four satellites in a tetrahedral configuration, the

Published by Copernicus GmbH on behalf of the European Geosciences Union. 


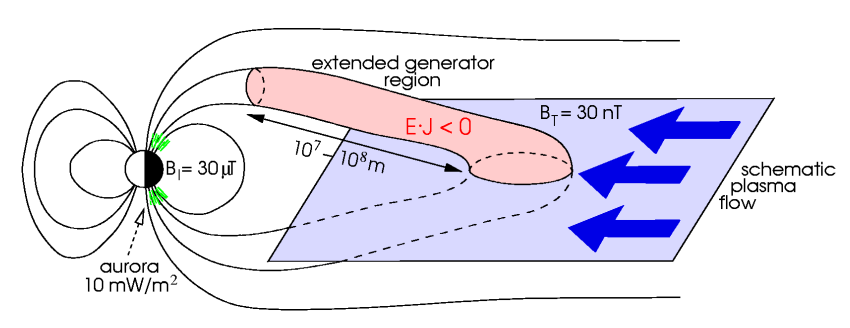

Fig. 1. Order of magnitude estimate for the power density in the generator region. In order to drive a moderately strong energy flux into the ionosphere, of $10 \mathrm{~mW} / \mathrm{m}^{2}$, a power density of $\sim 10^{-13}$ $10^{-12} \mathrm{~W} / \mathrm{m}^{3}$ is needed. We assume that the generator extends $\sim 1.5-15 R_{E}$ along the magnetic field line, and the flux tube cross section at generator level is $\sim 1000$ times larger than at ionospheric level.

complete determination of the current density becomes possible. In addition, each satellite is equipped with three instruments which are able to measure or to infer the electric field (EFW, EDI, and CIS), thus improving the reliability of the $\boldsymbol{E}$ estimates. At the same time, FAST offers an instantaneous view over the auroral electron precipitation and energy flux into the ionosphere, which helps to validate the $\boldsymbol{E} \cdot \boldsymbol{J}<0$ signatures seen by Cluster. In the following, we present a conjunction event, where Cluster crosses a region of $\boldsymbol{E} \cdot \boldsymbol{J}<0$ at the same time with the detection of accelerated electrons by FAST.

Our main goal will be to carefully check the experimental evidence and to prove that the $\boldsymbol{E} \cdot \boldsymbol{J}<0$ signature is real. In addition to producing evidence for the encounter of a generator region, we also aim to develop a method to be used in later studies addressing the auroral generator. In Sect. 2 we give an order of magnitude estimate for $\boldsymbol{E} \cdot \boldsymbol{J}$, and discuss the choice of the reference system. An overview of the instrumental setup is given in Sect. 3. The conjunction conditions and the data are introduced in Sects. 4 and 5, where we elaborate on those features proving that Cluster and FAST data are indeed conjugated. A thorough discussion of the $\boldsymbol{E} \cdot \boldsymbol{J}<0$ signature, of the generator region, and of its relationship to the auroral ionosphere, is given in Sect. 6.

Additional generator regions are presented and discussed in the companion paper, H06.

\section{A primer on the generator region}

\section{$2.1 \boldsymbol{E} \cdot \boldsymbol{J}$ estimate}

A moderately strong aurora is produced by $\mathrm{keV}$ precipitating particles, which carry an energy flux into the ionosphere of $\sim 10 \mathrm{~mW} / \mathrm{m}^{2}$ (the visibility threshold of $\sim 1 \mathrm{kR}$ corresponds to $\sim 1 \mathrm{~mW} / \mathrm{m}^{2}$ ). Assuming that the magnetic field in the auroral ionosphere and the outer magnetosphere is about $50 \mu \mathrm{T}$ and $50 \mathrm{nT}$, respectively, we obtain an area mapping factor of $\sim 1000$. Hence, by mapping the ionospheric energy flux to the distant magnetosphere, we obtain $\sim 10^{-5} \mathrm{~W} / \mathrm{m}^{2}$. Assuming that the extent of the generator region along the magnetic field line is in the range $10^{7}-10^{8} \mathrm{~m}(1.5-15$ Earth radii), we obtain a power density estimate in the generator region of $10^{-13} \mathrm{~W} / \mathrm{m}^{3} \lesssim \boldsymbol{E} \cdot \boldsymbol{J} \lesssim 10^{-12} \mathrm{~W} / \mathrm{m}^{3}$.

The diagram in Fig. 1 illustrates the calculation above. The estimated power density is quite small and consistent with electric fields and current densities of $\sim 1 \mathrm{mV} / \mathrm{m}$ and $\sim 1 \mathrm{nA} / \mathrm{m}^{2}$, respectively, which is close to the detection limits of the Cluster instruments. Obviously, one cannot expect to obtain high accuracy in evaluating $\boldsymbol{E} \cdot \boldsymbol{J}$. Nevertheless, by conjugate examination of high and low altitude data one can still obtain reliable information on the sign and trend of $\boldsymbol{E} \cdot \boldsymbol{J}$.

\subsection{Reference systems}

A correct choice of the reference frame is utterly important for the computation of $\boldsymbol{E} \cdot \boldsymbol{J}$. Although the current density is invariant under non-relativistic coordinate transformations, the electric field is not. By changing the reference frame one obtains different values, or even different signs, for the power density.

It makes sense to calculate $\boldsymbol{E} \cdot \boldsymbol{J}$ in the same reference frame as the loads, where the electromagnetic energy is converted into kinetic energy of the particles, heat and light. Two main load regions can be identified in the auroral current circuit: one is located at ionospheric level, where the fieldaligned currents close through Pedersen currents, which dissipate the electromagnetic energy by Joule heating, and the other load can be found in the auroral acceleration region, where the charges are accelerated by parallel electric fields, $E_{\|}$, to $\mathrm{keV}$ energies.

A suitable choice for the reference frame is the Geocentric Solar Ecliptic (GSE) system. The power consumption within the acceleration region, $E_{\|} J_{\|}$, is invariant under nonrelativistic transformations. On the other hand, the frame related to the Joule dissipation in the ionosphere is fixed to the neutral atmosphere, which moves with respect to GSE. However, this motion does not make a significant difference for the evaluation of $\boldsymbol{E} \cdot \boldsymbol{J}$. The velocity of the neutral atmosphere with respect to GSE, $\boldsymbol{v}_{N}$, is given by $\boldsymbol{v}_{N}=\boldsymbol{v}_{N E}+\boldsymbol{v}_{E}$, with $\boldsymbol{v}_{N E}$ the velocity of the neutral atmosphere with respect to the Earth (or neutral wind velocity) and $\boldsymbol{v}_{E}$ the velocity of the Earth's rotation. Since $v_{E} \simeq 0.5 \mathrm{~km} / \mathrm{s}$, while $v_{N E}$ at ionospheric $E$-layer altitudes (where the currents close) is not larger than a few $0.1 \mathrm{~km} / \mathrm{s}$ (Brekke et al., 1994; Nozawa and Brekke, 1995), $v_{N}$ is of the order of $1 \mathrm{~km} / \mathrm{s}$ or less. With a magnetic field of $30 \mathrm{nT}$, typical for the tail, such a motion implies a change in the electric field of less than $30 \mu \mathrm{V} / \mathrm{m}$, which is negligible when computing $\boldsymbol{E} \cdot \boldsymbol{J}$.

The Geocentric Solar Magnetospheric (GSM) system may seem to be more appropriate than GSE for investigating plasma sheet processes, because it naturally takes into account the symmetry changes induced by the rotation of the Earth's magnetic dipole. Although we shall use GSM for 
mapping purposes, we shall otherwise prefer GSE, because it is nearly identical to the Despun Satellite Inverted (DSI) system, the most convenient choice for the EFW electric field data (Sect. 3.1). DSI has its x-axis in the satellite spin plane, closest to the Sun direction, the z-axis is normal to the satellite spin plane, and the y-axis completes a right-handed system.

In addition, we shall also use a magnetic fieldaligned system, MAG, defined as follows: $\hat{x}=-\boldsymbol{B} / B$, $\hat{y}=\boldsymbol{V}_{\text {sat }} \times \boldsymbol{B} /\left|\boldsymbol{V}_{\text {sat }} \times \boldsymbol{B}\right|, \hat{z}=\hat{x} \times \hat{y}$, with $\hat{x}, \hat{y}, \hat{z}$ as unit vectors, $\boldsymbol{B}$ is the magnetic field, and $\boldsymbol{V}_{\text {sat }}$ is the satellite velocity; $\boldsymbol{B}$ and $\boldsymbol{V}_{\text {sat }}$ are averaged over the four Cluster spacecraft. The definition of MAG is tailored to its use in the southern plasma sheet, where the magnetic field and Cluster velocity point roughly in $-\hat{x}_{G S E}$ and $-\hat{z}_{G S E}$ direction, respectively, so that MAG is very close to GSE.

We note that GSE, GSM, DSI, and MAG are fixed with respect to each other and only the orientation of the axes is different. For the rest of the paper each time a direction, $x, y$, or $z$, is indicated, it will refer to GSE, if not explicitly stated otherwise.

\section{Instrumentation}

The four Cluster spacecraft were launched in July-August 2000 , in a high altitude polar orbit, with an inclination of $81^{\circ}$, an apogee at $18 R_{E}$, a perigee at $3 R_{E}$, and an orbital period of $57 \mathrm{~h}$. The satellites spin around axes nearly parallel to $\hat{z}_{G S E}$, with a 4-s period, and fly in a tetrahedral formation, whose scale size and shape can be adjusted according to the scientific goal.

The FAST satellite was launched in August 1996, in a rather low altitude polar orbit, with an inclination of $83^{\circ}$, an apogee at $4100 \mathrm{~km}$, a perigee at $400 \mathrm{~km}$, and an orbital period of $132 \mathrm{~min}$. The spacecraft spins every $5 \mathrm{~s}$ around an axis normal to the orbital plane. The magnetic field line is typically within a few degrees from the spin plane.

Both Cluster and FAST are equipped with complete sets of plasma and field instruments. Detailed descriptions of the payloads are given in special issues of Annales Geophysicae for Cluster (Escoubet et al., 2001, and references therein), and Space Science Reviews for FAST (Pfaff et al., 2001, and references therein). In the following we shall briefly review just those instrumental features important for our study.

\subsection{Cluster setup}

Data from three experiments on board Cluster can be used, in principle, to derive the DC electric field: the Electric Field and Wave experiment (EFW), the Electron Drift Instrument (EDI), and the Cluster Ion Spectrometer (CIS) - which itsself consists of two sensors, the Composition and Distribution Function Analyzer (CODIF) and the Hot Ion Analyzer (HIA). As discussed below, measuring the electric field in the plasma sheet represents a challenge for each instrument. One way to overcome the difficulties raised by the special plasma sheet conditions is to compare all the available electric field estimates and validate them just when they are reasonably similar for at least two instruments. Although such a criterion cannot fully guarantee the quality of the data, it increases the chance that the validated values are close to the real ones.

In the following we discuss in more detail the electric field data obtained from EFW, CIS/CODIF, and CIS/HIA, to be used in this study. Because of the low magnetic field and $\sim 1 \mathrm{keV}$ electron background, EDI (Paschmann et al., 2001) is usually not operational in electric field mode while Cluster crosses the plasma sheet. We also discuss the procedure used to obtain the current density from the data provided by the magnetic field experiment, FGM (Balogh et al., 2001).

\subsubsection{EFW electric field}

The EFW instrument (Gustafsson et al., 2001) consists of two pairs of spherical probes in the satellite spin plane. The potential differences between the paired probes can be used to find the instantaneous components of the electric field in the spin plane. If high resolution is not critical, as in our case, a better choice is to use electric field data averaged over one spin, available from each operational pair of probes. The advantage of using these data is that the electric field is less spiky, in particular the Sun and magnetic spikes are filtered out (the Sun/magnetic spike shows up each time the probe pair is aligned with the Sun/magnetic field direction). In addition to obtaining the electric field, the probes' potential information can also be used to infer the satellite potential, which represents a proxy for the plasma density.

In order to derive the full electric field vector from the EFW data, one assumes that the component parallel to the magnetic field vanishes, $E_{\|}=0$, which implies $\boldsymbol{E} \cdot \boldsymbol{B}=0$. Knowing the magnetic field vector and the electric field projection in the satellite spin plane, this equation can be solved to find the third electric field component, parallel to the spin axis. However, such an operation can be performed only when the magnetic field vector is not included in the spin plane. In practice, the angle between the magnetic field and the spin plane should be $\gtrsim 15^{\circ}$, a condition which is rarely met at $\sim 18 R_{E}$ in the plasma sheet, close to Cluster apogee. The magnetic field is pointing mainly in the $X$ direction, which is nearly parallel to the satellite spin plane. Hence, it is often not possible to derive the full electric field vector.

Another problem encountered by EFW in the plasma sheet is related to the low plasma density. Even if not as low as in the lobe, the density is low enough $\left(0.1-1 \mathrm{~cm}^{-3}\right)$ to result in large spacecraft potentials (up to $40 \mathrm{~V}$ ). When the Active Spacecraft Potential Control (ASPOC, Torkar et al., 2001) instrument is turned off and the spacecraft potential is high, the associated potential structure can seriously perturb the natural plasma environment, in particular the electric field. A comparative study of electric field data as yielded by EFW 
and EDI (Eriksson et al., 2006) has pointed out the key role of the spacecraft potential control in obtaining good $\boldsymbol{E}$ estimates from EFW.

Depending among others on the spacecraft potential, a sunward electric field offset of $\sim 1 \mathrm{mV} / \mathrm{m}$ can be present in the EFW data. In order to remove it, a manual calibration is needed. Because this offset is less critical for the computation of $\boldsymbol{E} \cdot \boldsymbol{J}$, we shall not enter into the details of this operation. In September the Cluster orbit is close to the noonmidnight meridian plane, so that the Sun direction is close to the $X$ axis and, for our tail event, also close to the direction of the magnetic field. Therefore, the solar offset is mainly present in $E_{\|}$, which is assumed to be negligible.

\subsubsection{CIS electric field}

The two sensors, CODIF and HIA, included in the CIS package (Rème et al., 2001), perform a comprehensive characterization of the ionic plasma component and are, to some extent, complementary. CODIF is a mass spectrometer, which consists of a top-hat electrostatic analyzer that separates the ions according to their energy per charge, and a time-of-flight section, which allows for the mass-per-charge determination. HIA only includes a top-hat electrostatic analyzer, and therefore it can only measure the energy per charge. In compensation, its counting electronics has a higher saturation threshold and HIA can accommodate larger fluxes than CODIF. Saturation, however, is not an item of concern in the plasma sheet.

The energy range, covered in logarithmically spaced steps, is $0.025-40 \mathrm{keV} / \mathrm{e}$ for CODIF and $0.005-30 \mathrm{keV} / \mathrm{e}$ for HIA. Both sensors can supply various data products, including onboard computed moments and 3-D distributions. The 3-D distributions from CODIF are collected for each major ion $\left(\mathrm{H}^{+}, \mathrm{O}^{+}, \mathrm{He}^{+}, \mathrm{He}^{++}\right)$and can be used for the off-line (ground) computation of the moments. The onboard moments are always delivered with a spin resolution, while the 3-D distributions can be accumulated over several spins. In this paper we shall use CODIF $\mathrm{H}^{+}$and HIA distributions, accumulated over 1 spin in $31 \times 88$ and $31 \times 128$ energy-angle bins, respectively. The CODIF/HIA 3-D data are delivered every second/third spin, which implies a time resolution of $8 \mathrm{~s} / 12 \mathrm{~s}$ for the respective ground moments.

In order to derive the electric field, we assume that the frozen-in condition holds, which implies that $\boldsymbol{E}$ can be computed from $\boldsymbol{E}=-\boldsymbol{V} \times \boldsymbol{B}$, once the velocity, $\boldsymbol{V}$, is known. The determination of $\boldsymbol{V}$ can be cross-checked by comparing the HIA and CODIF results. Because there are significant differences between the two sensors - which are actually two different instruments - one cannot expect to obtain identical results. However, as will be shown later in the paper, there is good agreement between the CODIF and HIA results. The differences originate mainly in the calibration of the instruments, in particular the evaluation of the detection efficiency.

A second cross-check can be performed by comparing the onboard and ground HIA values. This is possible just for the full energy range of the instrument, because the HIA onboard moments are only calculated for the full energy range. A similar cross-check is less relevant for CODIF: because of the complexity introduced by the mass discrimination, the calibration information is too voluminous and just a subset of it is stored on board, to be used for the online computation of the moments. Hence, for CODIF the ground moments are usually more reliable than the on board moments.

Apart from the problems related to the measurements, the computation of $\boldsymbol{E}$ by using ion velocity data raises two basic issues:

1. It is known that in a plasma that carries a current $\boldsymbol{J}$, the magnetic field is "frozen" in the electron fluid, which moves with a velocity $\boldsymbol{V}_{e}=\boldsymbol{V}-\boldsymbol{J} /$ ne (e.g. Alfvén and Fälthammar, 1963, p. 176). As we are particularly interested in finding $\boldsymbol{E} \cdot \boldsymbol{J}<0$ signatures, it may seem that what we need is $\boldsymbol{V}_{e}$, not $\boldsymbol{V}$. However, simple vector algebra shows that:

$$
\left(-\boldsymbol{V}_{e} \times \boldsymbol{B}\right) \cdot \boldsymbol{J}=[(-\boldsymbol{V}+\boldsymbol{J} / n e) \times \boldsymbol{J}] \cdot \boldsymbol{B}=(-\boldsymbol{V} \times \boldsymbol{B}) \cdot \boldsymbol{J} .
$$

Therefore, the power density estimate is not corrupted by using the ion velocity.

2. A certain amount of contamination of the ion drift with diamagnetic drift, $V_{d}=-\nabla \cdot \mathbf{P}_{\mathbf{i}} \times \boldsymbol{B} / n e B^{2}$, is, in principle, possible ( $\mathbf{P}_{\mathbf{i}}$ is the ion pressure tensor). In Sect. 5.2 we will show that such a contamination is not expected to play a major role in our case.

\subsubsection{Cluster current density}

The full current density vector can be derived on Cluster by using the Curlometer method (Robert et al., 1998a; Dunlop et al., 2002), which implements Ampère's law, $\boldsymbol{J}=\nabla \times \boldsymbol{B} / \mu_{0}$. It only probes currents on scales larger than the characteristic spacecraft separation, so that in order to resolve small spatial scales the Cluster tetrahedron must be as small as possible. On the other hand, the characteristic size should not be smaller than a few ion gyroradii, since in this case the ideal MHD conditions assumed to compute $\boldsymbol{E} \cdot \boldsymbol{J}$ do not hold any more. During our particular event the ion temperature does not exceed $4 \mathrm{keV}$, while the magnetic field is about $30 \mathrm{nT}$, which yields an ion gyroradius, $R_{g i} \leq 200 \mathrm{~km}$ (assuming that the ions are mostly protons). Since the spacecraft separation is about $1500 \mathrm{~km}$, the Cluster tetrahedron covers several ion gyroradii.

The shape of the tetrahedron is also important when applying the Curlometer. For reliable results, it is necessary that the satellite configuration is as close as possible to an equilateral tetrahedron. The shape of the tetrahedron is quantified by two parameters, the "planarity" and the "elongation", which are both 0 in the ideal case (Robert et al., 1998b). In our event, the Cluster elongation and planarity are 0.08 and 
0.1 , respectively, a rather optimal situation to apply the Curlometer.

A rough quality indicator for the estimated current density is provided by $\epsilon_{B}=\nabla \cdot \boldsymbol{B} /|\nabla \times \boldsymbol{B}|$. Although in theory this quantity should be identically zero, in practice it can vary substantially, mainly due to nonlinearities in the magnetic field. Measurement errors and the orientation and shape of the tetrahedron can also contribute to this quantity. However, it should be noted that there exists no one-to-one correspondence between the value of $\epsilon_{B}$ and the actual error in the estimated current. As will be shown later in the paper, it can happen that $\epsilon_{B}$ is substantial even if the current provided by the Curlometer is consistent with the physical expectations.

\subsection{FAST setup}

The FAST payload includes electron and ion spectrometers, a mass spectrometer, DC and AC magnetometers, as well as several pairs of spherical probes to measure the electric field in various frequency ranges. In this study we shall use mainly electron data, as well as additional ion, composition, and DC magnetic field information; electric field data were not available.

Both the electron (EESA) and ion (IESA) spectrometers (Carlson et al., 2001) consist of two electrostatic sensors located on opposite sides of the spacecraft - each of them covering a $180^{\circ}$ field-of-view in the spin plane. As already mentioned, the magnetic field is always within a few degrees from the spin plane, and additional deflection plates can adjust the spectrometers' viewing direction, so that the magnetic field line is included in this plane. As a result, distribution functions covering the complete pitch-angle range, divided in 32 angular bins, can be sampled at a very high rate (as high as every $78 \mathrm{~ms}$, when the satellite is in Burst mode). During the event presented in this study FAST was in Slow Survey mode, implying a time resolution of $2.5 \mathrm{~s}$ (32 energy sweeps per distribution). The energy range, extending from $4 \mathrm{eV} / \mathrm{e}$ to $30 \mathrm{keV} / \mathrm{e}$ for EESA and from $3 \mathrm{eV} / \mathrm{e}$ to $25 \mathrm{keV} / \mathrm{e}$ for IESA, is divided into 48 logarithmically spaced bins.

The time of flight energy angle mass spectrometer (TEAMS, Klumpar et al., 2001) is in many respects similar to CODIF. It can resolve the 3-D distributions of the major ionospheric ions $\left(\mathrm{H}^{+}, \mathrm{O}^{+}, \mathrm{He}^{+}\right)$, sampling them in $48 \mathrm{log}$ arithmic energy bins, between $1 \mathrm{eV} / \mathrm{e}$ and $12 \mathrm{keV} / \mathrm{e}$, and 64 solid angle bins. TEAMS uses the spacecraft spin to acquire the 3-D distribution functions; its highest time resolution is $2.5 \mathrm{~s}$ for $\mathrm{H}^{+}$and $\mathrm{O}^{+}$, and $5 \mathrm{~s}$ for $\mathrm{He}^{+}$. During our conjunction event $\mathrm{H}^{+}$and $\mathrm{O}^{+}$were accumulated over $20 \mathrm{~s}$, and $\mathrm{He}^{+}$ over $40 \mathrm{~s}$.

The DC and low frequency magnetic field is measured with a tri-axes fluxgate magnetometer (Elphic et al., 2001). The satellite is magnetically clean, which allows for high data accuracy. The magnetometer range extends from $-65000 \mathrm{nT}$ to $65000 \mathrm{nT}$, thus covering completely the background Earth field, with a resolution of $\pm 2 \mathrm{nT}$. The mag-

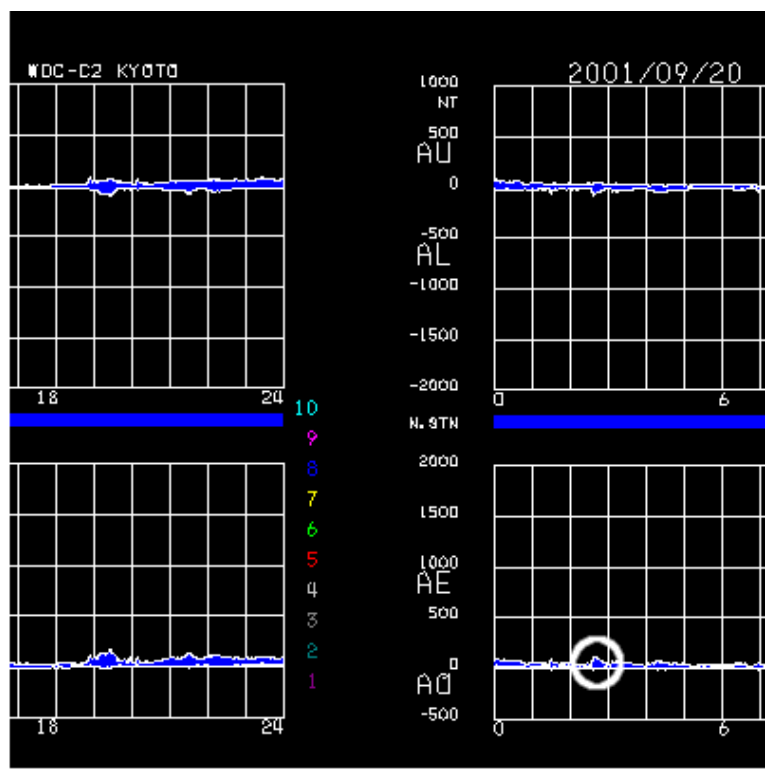

Fig. 2. Auroral activity indices, from the Data Service of World Data Center for Geomagnetism, Kyoto, http://swdcdb.kugi.kyoto-u. ac.jp (adapted). A small bump in AE, at the time of the conjunction, is indicated by a small circle. Its possible relevance is discussed in Sect. 5.1.

netic perturbation in the auroral zone, produced by FACs and/or Alfvén waves, is obtained by subtracting a model field (IGRF2000 in our case) from the measured data.

\section{Conjunction conditions}

A query to SSCWeb (http://sscweb.gsfc.nasa.gov) on FASTCluster conjunctions results in a large pool of hundreds of events. Among them, particularly interesting are the series of conjunctions which take place on consecutive FAST orbits. Because the inclinations of the FAST and Cluster orbital planes are almost identical, the precession of the FAST orbit in the inertial space by $-0.5^{\circ}$ /day ensures that every $\sim 1$ year the orbital planes are very close to each other, which leads to an increased conjunction rate. Such a favorable time interval was September-October 2001, when the conjunctions took place in the evening-pre-midnight Southern Hemisphere, with FAST close to apogee above the southern auroral oval and Cluster in the plasma sheet.

We have selected a sequence of three conjunctions, $\mathrm{CJ} 1-\mathrm{CJ} 3$, at 22:23, 00:29, and 02:36 UT, respectively, on 19-20 September 2001. In this section we give an overview of the entire interval. Later in the paper we shall concentrate on CJ3, while CJ1 and CJ2 are investigated in H06.

The magnetosphere was quiet during this time, with $K_{p}=1$. As illustrated by the $A E$ index in Fig. 2, the auroral activity was reduced as well (one notes, however, a small bump at CJ3 time, which provides a clue for the interpretation of the FAST data - see Sect. 5.1). Under such 


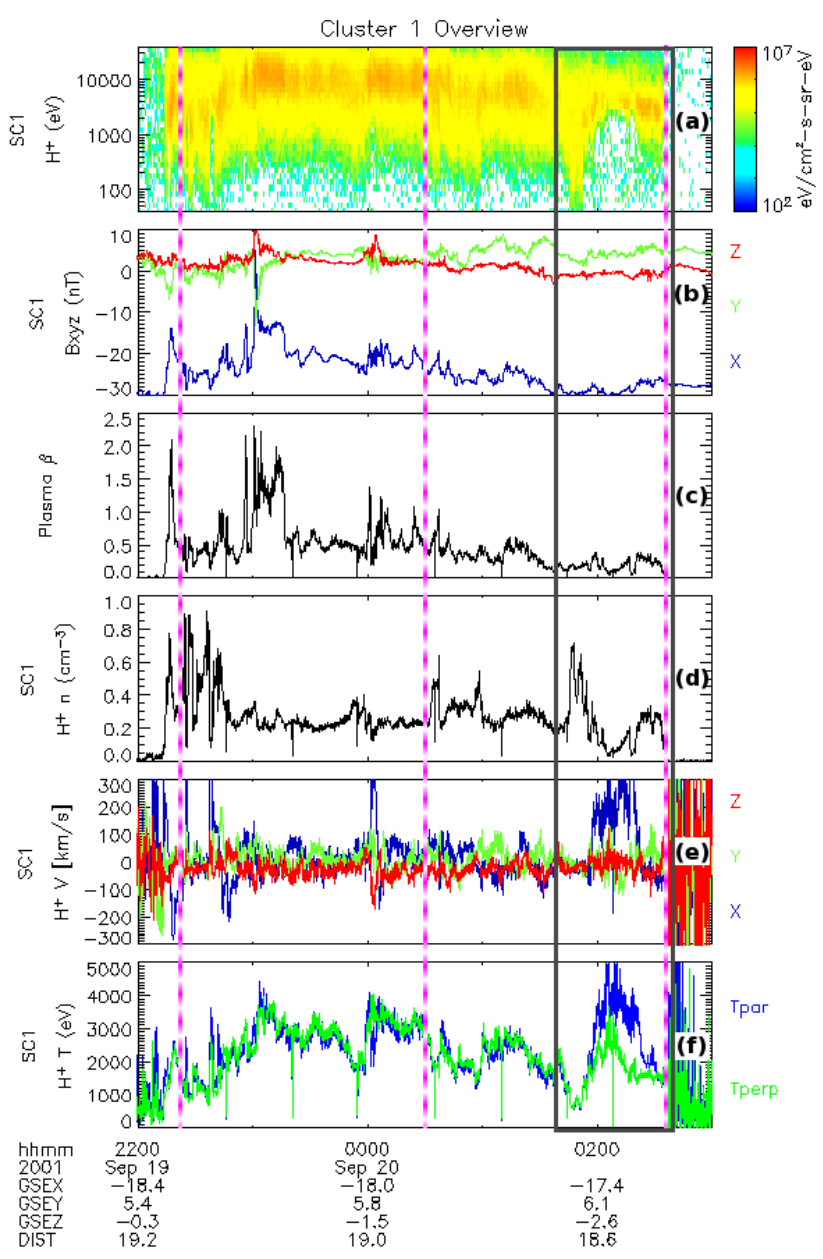

Fig. 3. Overview of $5 \mathrm{~h}$ of Cluster 1 data, measured in the southern plasma sheet. From top to bottom: (a) CIS/CODIF proton energy spectrogram. (b) Magnetic field. (c) Plasma $\beta$, equal to the ratio of proton to magnetic pressure. (d-f) Plasma density, velocity, and temperature, as derived from the proton data. The conjunction times are indicated by magenta vertical lines. The rectangle shows the interval investigated in this paper. The interval 22:00-01:30 UT is discussed in H06.

quiet conditions the mapping along the magnetic field line is more reliable and the various disturbance factors, like inductive electric fields (e.g. Paschmann et al., 2003, p. 248), are less prone to perturb the model configuration. Therefore, one can expect the conjunction geometry and timing, as obtained by the T96 magnetic field model (Tsyganenko, 1995), to be reasonably accurate.

Cluster entered the northern plasma sheet on 19 September at about 12:45 UT, and crossed the neutral sheet around 16:00 UT. Because of the plasma sheet motion, the spacecraft made an excursion to the southern lobe from 20:45 UT to 22:15 UT, and then returned to the plasma sheet until about 02:36 UT. Figure 3 shows an overview of Cluster 1 data for the interval 22:00-03:00 UT. The boundaries of the plasma sheet encounter are clearly visible in the energy spectrogram (a). Since the $x$ component of the magnetic field stays negative (b), mostly between about -30 and $-20 \mathrm{nT}$, the satellite remains below the neutral sheet, most of the time not very far from the lobe boundary. The deepest penetration towards the central plasma sheet is observed around 23:00 UT, when the plasma $\beta$ parameter (c) reaches values larger than 2 .

The overview is completed by showing the proton density (d), velocity (e), and temperature (f), derived by taking the moments of the CODIF 3-D distributions. The density has typical plasma sheet values, between 0.1 and $1 \mathrm{~cm}^{-3}$. The velocity stays most of the time below $100 \mathrm{~km} / \mathrm{s}$, with components of a few $10 \mathrm{~km} / \mathrm{s}$. An outstanding feature is the presence of the $\sim 300 \mathrm{~km} / \mathrm{s}$ peaks in $V_{x}$, near both ends of the plasma sheet interval, but also inside it, shortly after 00:00 UT. In Sect. 5.2 we shall examine in more detail the broader velocity peak around 02:10. At the same time with this peak, but extending to the very limit of the plasma sheet, the temperature shows a substantial anisotropy, which is further discussed in Sect. 6.2. For the rest of the time the temperature is mostly isotropic and takes typical plasma sheet values, between 1 and $4 \mathrm{keV}$. Note that the downward spikes, best visible in the temperature panel, are instrumental artifacts produced by accumulation drop-outs in the $>1 \mathrm{keV}$ CODIF bins.

The ionospheric footprints of Cluster 1 (nearest conjunction) and FAST, at 110-km altitude, are shown in the top plot of Fig. 4, in eccentric dipole coordinates. The Cluster 1 trace corresponds to 5h, 22:00-03:00 UT, while for FAST only $4 \mathrm{~min}$ around the CJ3 time are shown. At the conjunction time the spacecraft are at the same magnetic latitude and separated by $\sim 30 \mathrm{~min}$ in magnetic local time. The footprints are located above Antarctica, so that conjugate ground information - magnetic, radar, or optical - is not available. Alike, there is no additional optical data from the IMAGE or Polar satellites.

A complementary view on the conjunction is offered by the bottom plot in Fig. 4, which shows, for the same time intervals, the tail footprint of FAST, together with the projection of Cluster 1 in the $(Y, Z)_{G S M}$ plane. At the conjunction time the spacecraft are separated by $\sim 1.5 R_{E}$ in the $Y$ direction, consistent with the local time separation in the ionospheric projection. This distance is substantially larger than the scale size of the Cluster tetrahedron $(\sim 1500 \mathrm{~km})$ and the ion gyroradius $(\lesssim 200 \mathrm{~km})$, which indicates that FAST and Cluster are not very closely conjugated. However, the separation between the respective flux tubes is tolerable, given the tendency towards azimuthal symmetry within the auroral oval. Incidentally, we note that the respective distances between the tail and ionospheric projections of Cluster and FAST provide an estimate for the linear mapping factor in the $Y(\mathrm{E}-\mathrm{W})$ direction of $\sim 35$. As the surface mapping factor is $\sim 1700$ (equal to the magnetic field ratio between the ionosphere and Cluster), the linear mapping factor in the $Z(\mathrm{~N}-\mathrm{S})$ direction is $\sim 50$. 
In performing the mapping along the magnetic field line we used the IGRF2000 model for the internal field and the T96 model for the external field. The solar wind parameters needed by $\mathrm{T} 96$ had small variations around $P_{s w}=1.7 \mathrm{nPa}$, $B_{y}=5 \mathrm{nT}$, and $B_{z}=1 \mathrm{nT}$, while the Dst index stayed between 0 and 4. An alternative model to T96 is the upgraded T01 (Tsyganenko, 2002a,b), which takes into account the magnetospheric memory by including two new parameters that quantify the solar wind state one hour before the actual time. However, in our quiet case, with little variation in the solar wind, T01 is not expected to bring substantial changes. In addition, T01 is based on data collected within $15 R_{E}$ from the Earth, while Cluster is a little more distant.

For the rest of this paper we shall focus our attention on $\mathrm{CJ} 3$ and, as far as Cluster is concerned, on the 1-h preceding it. A detailed analysis of $\mathrm{CJ} 1$ and $\mathrm{CJ} 2$, with more emphasis on the overall picture, is presented in H06. One notes that $\mathrm{CJ} 3$ is located at the very boundary between the plasma sheet and the lobe, which makes it easier to cross-check the magnetic field line mapping by the morphology of the particle precipitation. In addition, close to a boundary in the plasma there are good chances of finding the ingredients needed for a generator region, that is electrical currents and motion coupled electric fields (to be discussed further in Sect. 6.2).

\section{Data}

When close to their respective apogees, Cluster and FAST have different velocities ( $\sim 1 \mathrm{~km} / \mathrm{s}$ and $\sim 6 \mathrm{~km} / \mathrm{s}$, respectively) and the flux tube sections are widely different. Consequently, the same latitude interval in the ionospheric projection is covered by FAST in a few minutes and by Cluster in a few hours. Therefore, we shall present just a few minutes of FAST data, with emphasis on 2 min close to conjunction time, but use $1 \mathrm{~h}$ of Cluster data. Because the magnetosphere is dynamic even at "quiet" times, we do not expect, however, that the data conjugated in space can be related, except for those data which are also reasonably well conjugated in time.

\subsection{FAST data}

Figure 5 presents data measured by FAST during its pass over the evening side of the southern auroral oval. In order to give a broader perspective, the upper part of Fig. 5 shows the magnetic field (a), electron (b-e), and ion ( $f, g)$ data, during a longer time interval, encompassing the entire crossing above the oval. In the following we shall concentrate only on the segment 02:35:50-02:37:50, near the conjunction, when FAST was in the vicinity of the polar cap boundary (PCB).

In the lower part of Fig. 5 we zoom in on the magnetic field (h) and electron (i, j) data collected between 02:35:50 and $02: 37: 10$, the interval which we believe is directly associated with the generator region crossed by Cluster (Sect. 5.2). During this interval the electron energy (b) and pitch-angle
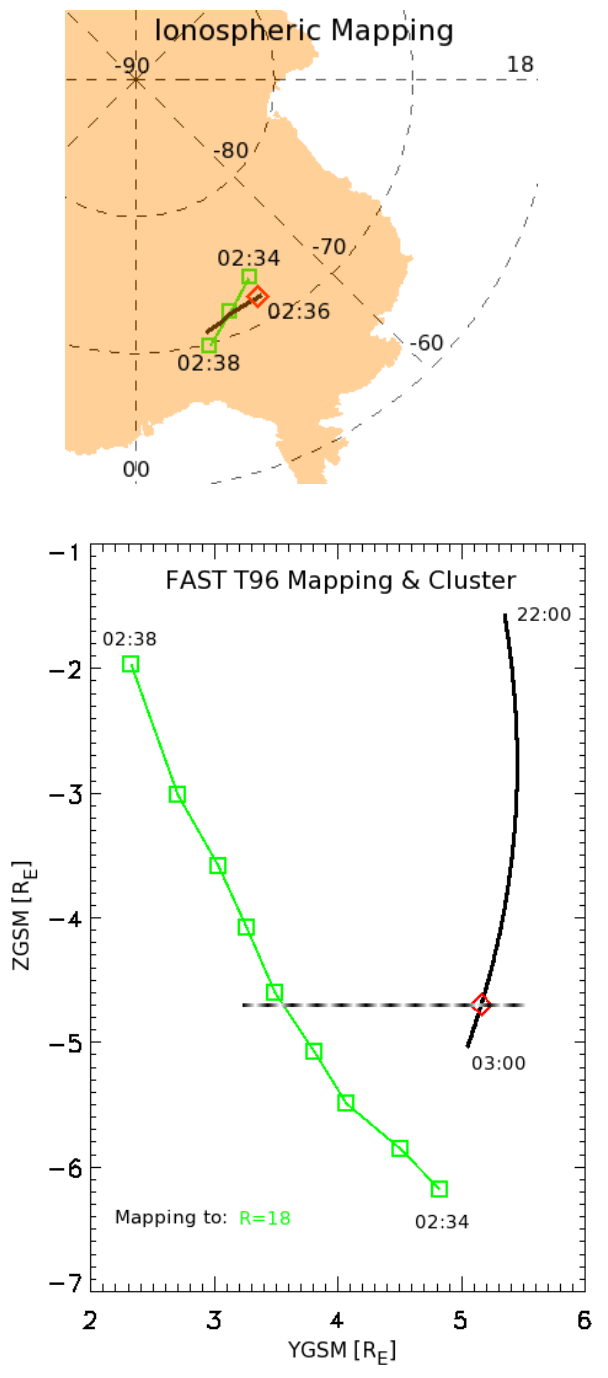

Fig. 4. Top: Ionospheric projections of Cluster 1 and FAST in eccentric dipole coordinates. Midnight is at the bottom and dusk to the right (Southern Hemisphere). The FAST footprint is shown 4 min around CJ3 time, while for Cluster 1 we have the same 5-h interval as in Fig. 3. Bottom: Tail projections of FAST and Cluster 1 in $\left(Y_{G S M}, Z_{G S M}\right)$ coordinates. The FAST position is mapped to $18 R_{E}$, about equal to the geocentric distance of Cluster around the conjunction time. In both plots FAST is indicated with green squares while the Cluster 1 trace is shown in black and a red diamond is overplotted at the conjunction time.

(c) spectrograms show an accelerated, $\sim 1.5 \mathrm{keV}$ population, pretty much isotropic outside the loss-cone - which indicates energization by quasi-stationary parallel electric fields, high (few $1000 \mathrm{~km}$ ) above the satellite. At the same time, the ion energy (f) and pitch-angle (g) spectrograms exhibit an energetic loss-cone population of plasma sheet origin.

As seen in the panels (i) and (j), the electrons carry net number and energy fluxes into the ionosphere, $\Phi_{N} \lesssim 10^{8} \# / \mathrm{cm}^{2} \mathrm{~s}$ and $\Phi_{W} \lesssim 0.25 \mathrm{~mW} / \mathrm{m}^{2}$. When mapped to 


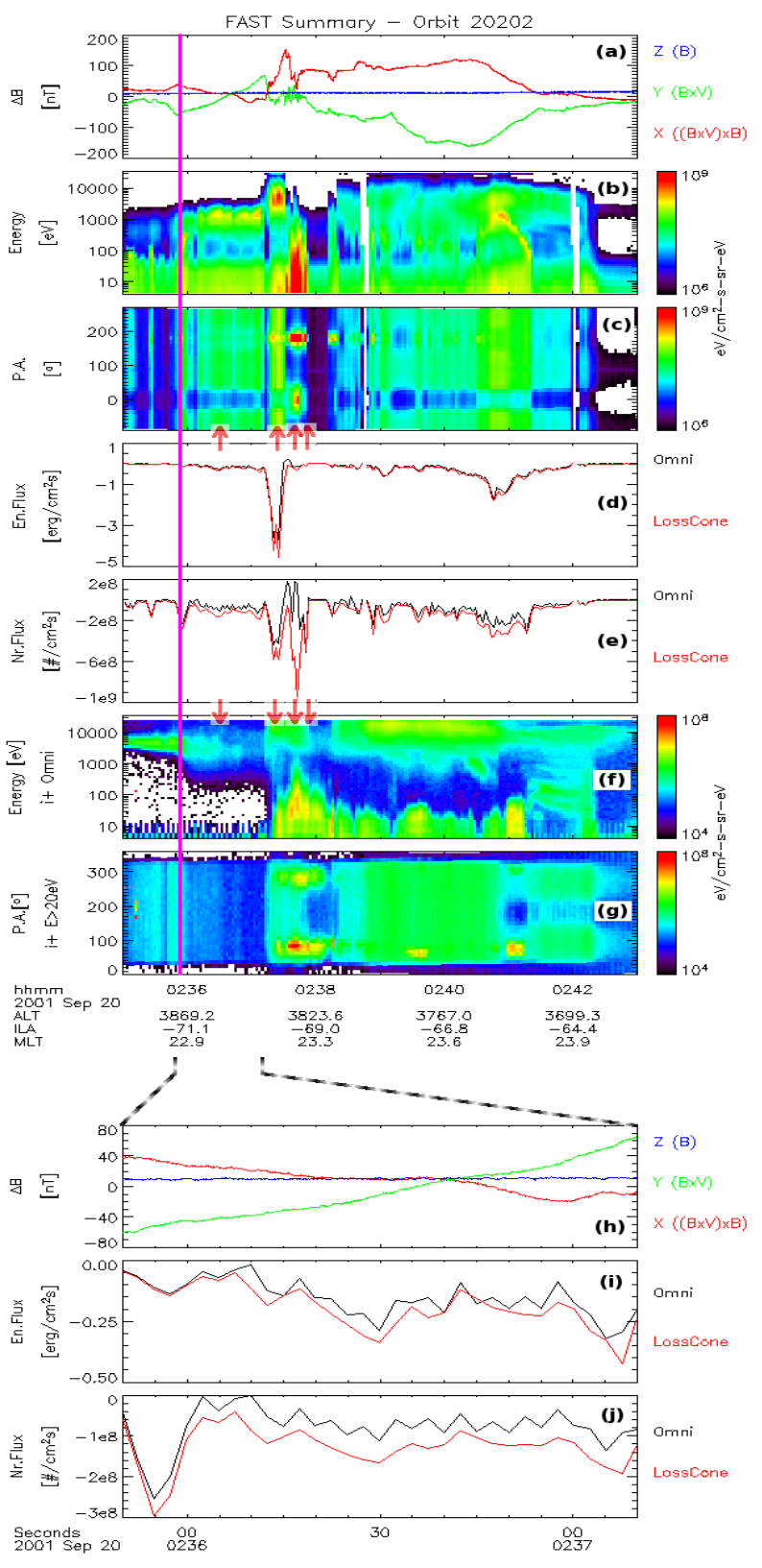

Fig. 5. Top: summary of FAST data: Magnetic field perturbation (a), electron data (b-e), and ion data (f, g). The electron panels show: EESA energy and pitch-angle spectrograms (b, c), energy and number flux (d, e). The energy and number fluxes are obtained by summing over electrons above $50 \mathrm{eV}$, omnidirectional (black) and within the loss-cone (red). For the ions we show IESA energy and pitch-angle spectrograms (f, g). The vertical magenta line indicates the conjunction with Cluster, while the arrows below panel (c) and above panel (f) indicate the times of the distribution functions in Fig. 6. Bottom: zoom in on $80 \mathrm{~s}$ of data near the polar cap: Magnetic field perturbation (h), energy flux (i), and number flux (j). These data are reasonably consistent with a current sheet geometry, almost parallel (within $\sim 15^{\circ}$ ) to the magnetic $\mathrm{E}-\mathrm{W}$ direction. the ionosphere, the energy flux becomes $\Phi_{W}^{I} \lesssim 1 \mathrm{~mW} / \mathrm{m}^{2}$, which corresponds roughly to the visibility limit.

The relatively uniform variation in the $X$ and $Y$ components of the perturbation magnetic field, $\Delta B(\mathrm{~h})$, suggests an arc-like geometry for the faint auroral form overpassed by FAST. The changes in $\Delta B_{Y}$ and $\Delta B_{X}$ are $\delta_{\Delta B_{Y}} \simeq 100 \mathrm{nT}$ and $\delta_{\Delta B_{X}} \simeq-60 \mathrm{nT}$, respectively. Assuming infinite elongation, one can find the angle $\theta$ between the normal to the arc and the satellite footprint: $\tan \theta=\left|\delta_{\triangle B_{X}}\right| / \delta_{\Delta B_{Y}} \simeq 0.6 \Rightarrow \theta \simeq 30^{\circ}$. Since the FAST footprint makes an angle of $\sim 45^{\circ}$ with the magnetic latitude circles (top of Fig. 4a), it follows that the arc is almost aligned (within $\sim 15^{\circ}$ ) in the magnetic E-W direction, consistent with the expectations for a quiet arc.

The magnetic field information can also be used to compute the FAC density, $j_{\|}^{b}$, inside the arc, by $j_{\|} \simeq \delta_{\Delta B} / \mu_{0} d$, with $\delta_{\triangle B}=\sqrt{\delta_{\Delta B_{X}}^{2}+\delta_{\Delta B_{Y}}^{2}} \simeq 120 \mathrm{nT}$ and $d$ as the arc thickness. One can find $d$ from $d=L_{S} \cos \theta=V_{S} \tau \cos \theta$, where $L_{S}$ is the length of the satellite path across the arc, $V_{S}$ is the satellite velocity, and $\tau$ is the time needed to cross the arc. With $V_{S} \simeq 5.6 \mathrm{~km} / \mathrm{s}$ and $\tau \simeq 80 \mathrm{~s}$ we find $d \simeq 400 \mathrm{~km}$ and $j_{\|}^{b} \simeq 0.24 \mu \mathrm{A} / \mathrm{m}^{2}$. This value compares reasonably well to the one obtained by using the electron flux, $j_{\|}^{e}=e \Phi_{N} \lesssim 0.16 \mu \mathrm{A} / \mathrm{m}^{2}$. The difference between $j_{\|}^{b}$ and $j_{\|}^{e}$ can be traced back to low energy electrons, not detected by EESA, and to deviations from the infinite arc approximation.

From 02:37:10 to $02: 37: 50$ there is a dramatic change in the FAST data: the magnetic field exhibits a complicated variation in both $X$ and $Y$ components, no longer compatible with a quiet arc; the electrons show a strong and narrow increase in the energy flux (d), up to $4.5 \mathrm{~mW} / \mathrm{m}^{2}\left(18 \mathrm{~mW} / \mathrm{m}^{2}\right.$ mapped to ionosphere, implying a bright aurora), followed by a region of less energetic but intense counterstreaming fluxes (e); the ions exhibit a substantial ionospheric component, rich in oxygen (not shown) and transversely accelerated up to more than $100 \mathrm{eV}$.

By using the data from one satellite, with no additional optical information, it is not possible to resolve the spatial versus temporal ambiguity. Therefore, we cannot decide beyond any doubt on the nature of the change in the FAST data around $02: 37: 10$. However, we believe that the temporal variability plays the prime role. The change in the data can be associated with the bump in the $A E$ index, emphasized in Fig. 2, and this indicates that FAST captures the onset of a small substorm. What may be a bit of a surprise is that the intense electron precipitation related to the peak in the energy flux, which presumably induced a significant ionospheric current, caused just a small $A E$ variation. One should not forget, however, that the $A E$ index is mainly based on data collected in the Northern Hemisphere, which is not necessarily in a one-to-one relationship with the Southern Hemisphere. 

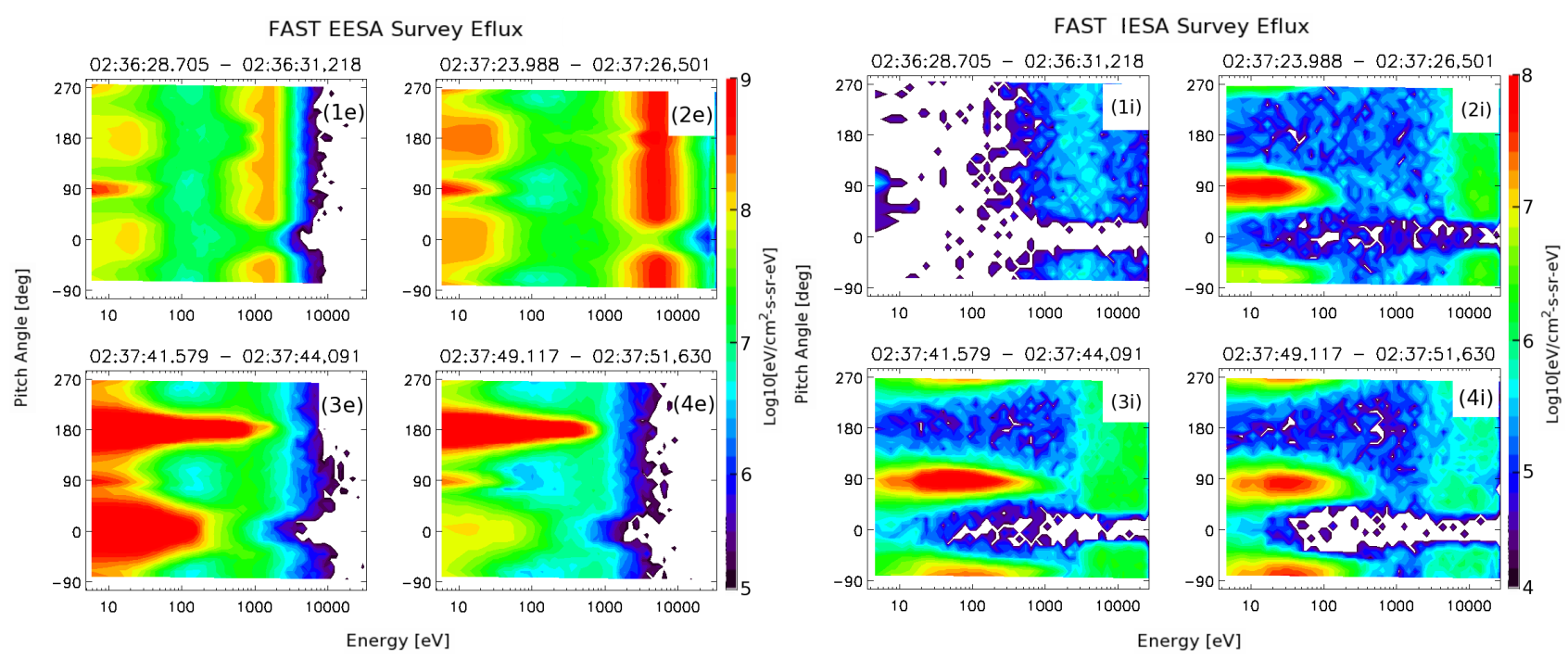

Fig. 6. Selection of FAST electron (left) and ion (right) distribution functions, in energy - pitch-angle coordinates. The respective times are indicated by arrows in Fig. 5, and were chosen as follows: at the middle of the less energetic electron precipitation interval (1), at the maximum electron energy flux (2), at the maximum electron upward (3) and downward (4) number flux.

In order to complete the view over the FAST data, in Fig. 6 we present a selection of electron and ion distribution functions, measured between 02:35:50 and 02:37:50, at the times indicated by arrows in Fig. 5. The distributions $(1 \mathrm{e}, 1 \mathrm{i})$ are typical for the quiet interval 02:35:50-02:37:10 and show $\sim 1.5 \mathrm{keV}$ accelerated electrons, together with losscone plasma sheet ions. The low energy electron component consists mainly of photoelectrons and secondaries, originating at the spacecraft. There are no low energy ionospheric ions.

The distributions (2), (3), and (4) are collected during the active interval 02:37:10-02:37:50. Besides the loss-cone plasma sheet ions, the transversely accelerated ionospheric component is now clearly visible in the ion frames. The asymmetry between $90^{\circ}$ and $270^{\circ}$, better seen in frame (2i), is probably caused by the ram velocity of the dominant oxygen ions. The electrons in frame (2e), corresponding to the maximum energy flux, are also accelerated, as in frame (1e), but have considerably higher energies and fluxes. The electron distributions (3e) and (4e) exhibit different features. In both cases counterstreaming fluxes are present, more prominently in frame (3e). In addition, the electrons are substantially heated, indicating wave-particle interactions.

Most probable, the heating of both the electrons and the ions has its primary origin in Alfvénic activity, suggested to have taken place by the small-scale structure in $\Delta B_{Y}$ (panel (a) in Fig. 5). Kinetic Alfvén waves can accelerate the electrons, which, in turn, can generate higher frequency waves, able to heat the ions. Although we cannot perform a rigorous check, because of missing electric field data, this is consistent with the substorm onset, when Poynting flux is exchanged back and forth between the magnetosphere and the ionosphere by means of Alfvén waves. Such an exchange is often located in the vicinity of the PCB (e.g. Wygant et al., 2000), within the so-called "Alfvénic region" (Paschmann et al., 2003, p. 94 and 195). In the electron energy spectrogram (panel (b) in Fig. 5) this region appears to be separated from the PCB by the quiet arc. However, since the FAST footprint is inclined at $45^{\circ}$ with respect to the magnetic latitude circles (Fig. 4a), the Alfvénic region could still be located at the PCB. This would require that the PCB goes down to lower latitudes towards midnight, a feature in agreement with the standard behavior of the auroral oval (e.g. Holzworth and Meng, 1975).

\subsection{Cluster data}

A summary of the data collected by Cluster between 01:40 UT and 02:40 UT, most of which is required in the evaluation of the power density, is presented in Fig. 7. The $\mathrm{SC} 1$ proton energy spectrogram in the top panel provides a condensed view over the particle context. The other panels show the proton velocity (b), the magnetic field (c), the ratio $\nabla \cdot \boldsymbol{B} /|\nabla \times \boldsymbol{B}|(\mathrm{d})$, the electric field and current (e-g), as well as the power density (h) and its cumulative sum (i). The magnetic field is averaged over all spacecraft, while the velocity and electric field are averaged over SC 1, 3, and 4, where CODIF data are available. The most prominent features in Fig. 7 are the high $V_{x}$ velocity towards the Earth, between 01:57 and 02:22 (panel b), and the negative variation in the cumulative sum of $\boldsymbol{E} \cdot \boldsymbol{J}$, between 22:17 and 22:30 (panel i).

The quantities presented in Fig. 7 are not only spatial averages over the spacecraft, but also temporal averages over $24 \mathrm{~s}$, which is roughly the time needed by the plasma to cross 


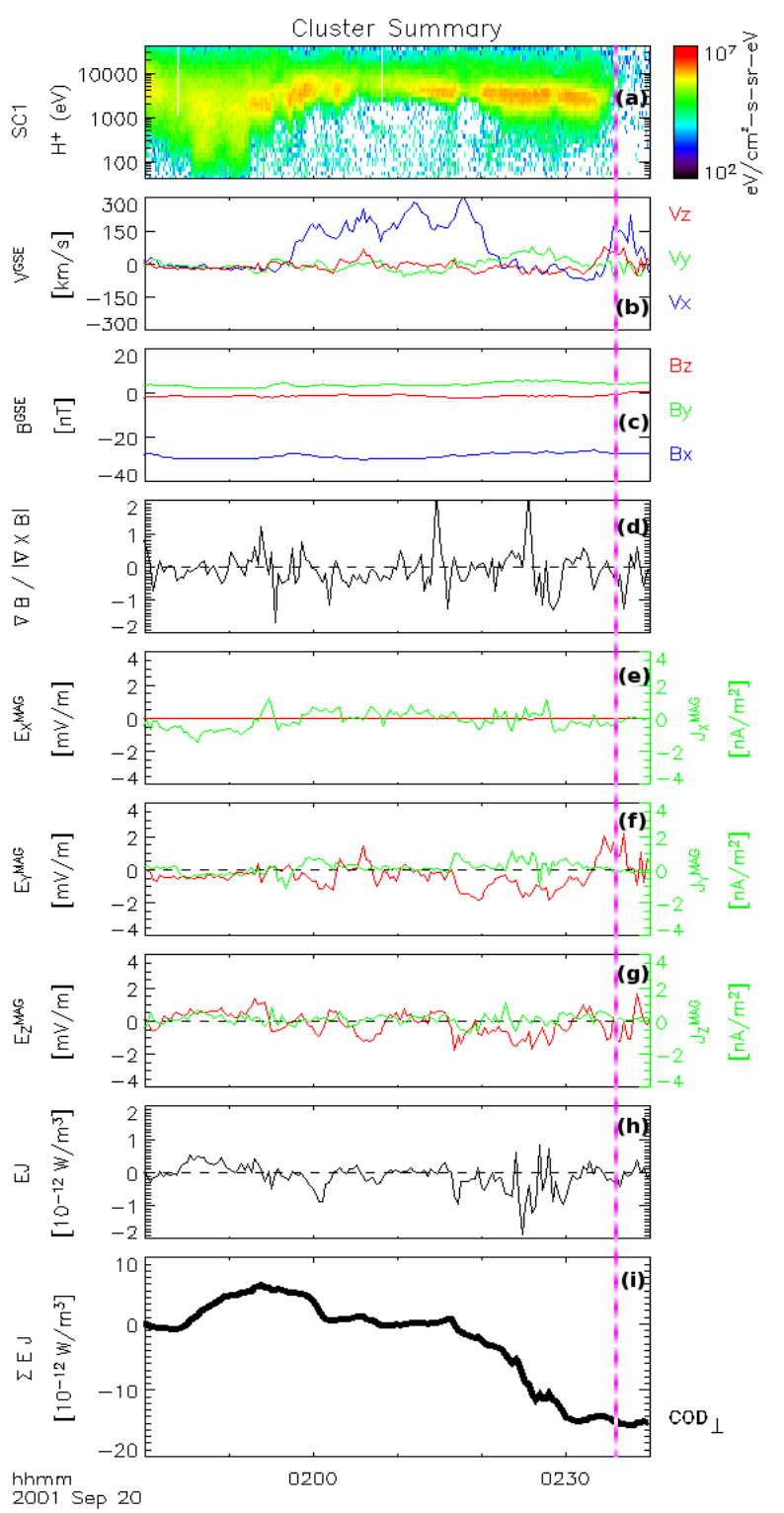

Fig. 7. Summary of Cluster data for the time interval 01:40-02:40: (a) CODIF $\mathrm{H}^{+}$energy spectrogram from $\mathrm{SC}$; ; average (b) $\mathrm{H}^{+}$velocity and (c) magnetic field; (d) normalized divergence of the magnetic field, $\nabla \cdot \boldsymbol{B} /|\nabla \times \boldsymbol{B}| ;(\mathbf{e}-\mathbf{g})$ components of the electric field, as derived from CODIF data, and current density, in the MAG reference system; (h) $\boldsymbol{E} \cdot \boldsymbol{J}$ and (i) its cumulative sum. The velocity, as well as the electric field, are averaged over SC1, SC3, and SC4. The magnetic field is averaged over all spacecraft. The conjunction with FAST is indicated by the vertical magenta line.

the Cluster tetrahedron (for velocities of $\sim 50 \mathrm{~km} / \mathrm{s}$ ). This choice is motivated as follows: by using the Curlometer one obtains a current which is representative for the entire tetrahedron, and can be best associated with the tetrahedron mass center. On the other hand, the electric field is derived by using one-satellite information and is therefore associated with

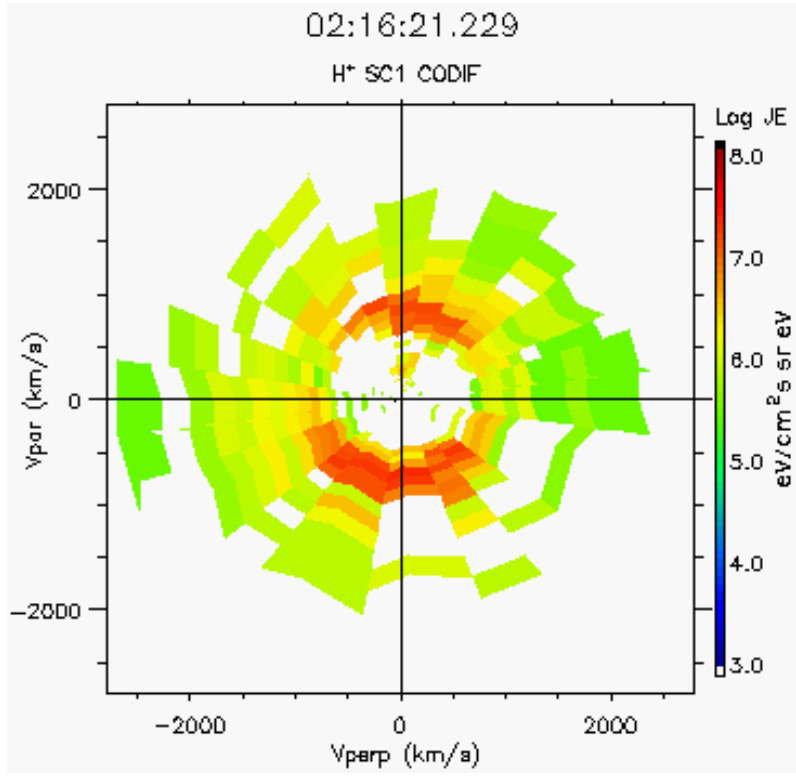

Fig. 8. Distribution function measured by CODIF in the PSBL. The $V_{\text {par }}$ axis is along the magnetic field while $V_{\text {perp }}$ is included in the plane defined by the magnetic field and the flow velocity. One notes the presence of $\sim 3 \mathrm{keV}$ ions streaming to and from the Earth, a typical PSBL feature.

the vertices of the tetrahedron. By averaging over the time needed by the plasma to cross the tetrahedron, one obtains $\boldsymbol{E}$ and $\boldsymbol{J}$ values representative for about the same plasma volume.

One issue of concern in Fig. 7 is the ratio $\epsilon_{B}=\nabla \cdot \boldsymbol{B} /|\nabla \times \boldsymbol{B}|$, which is considered to provide an indirect estimate for the error in the evaluation of the current. As seen in panel (d), $\epsilon_{B}$ is quite large at the time when the generator signature is detected. This issue is addressed in more detail in the next section, where we show that the current is nonetheless consistent with the expectations near the plasma sheet boundary.

The velocity of the plasma in panel (b) of Fig. 7 exhibits a substantial flow towards the Earth, of $150 \mathrm{~km} / \mathrm{s}$ to $300 \mathrm{~km} / \mathrm{s}$, between 01:57 and 02:22. A more detailed view on the proton data collected by CODIF within this earthward flow region is offered by Fig. 8, which shows a typical distribution, projected onto the plane, defined by the directions of the magnetic field and of the flow velocity. One notes the protons streaming earthward (negative $V_{p a r}$ ) and tailward, with peaks around the magnetic field direction at velocities around $750 \mathrm{~km} / \mathrm{s}$. This is a typical feature for the PSBL, where plasma supposed to originate from a reconnection site down the tail streams towards the Earth, along the magnetic field lines, up to the point where it is reflected back to the tail by the mirror force. The streaming protons are encountered within some distance from the boundary of the plasma sheet, which can be explained by the convection of the magnetic 

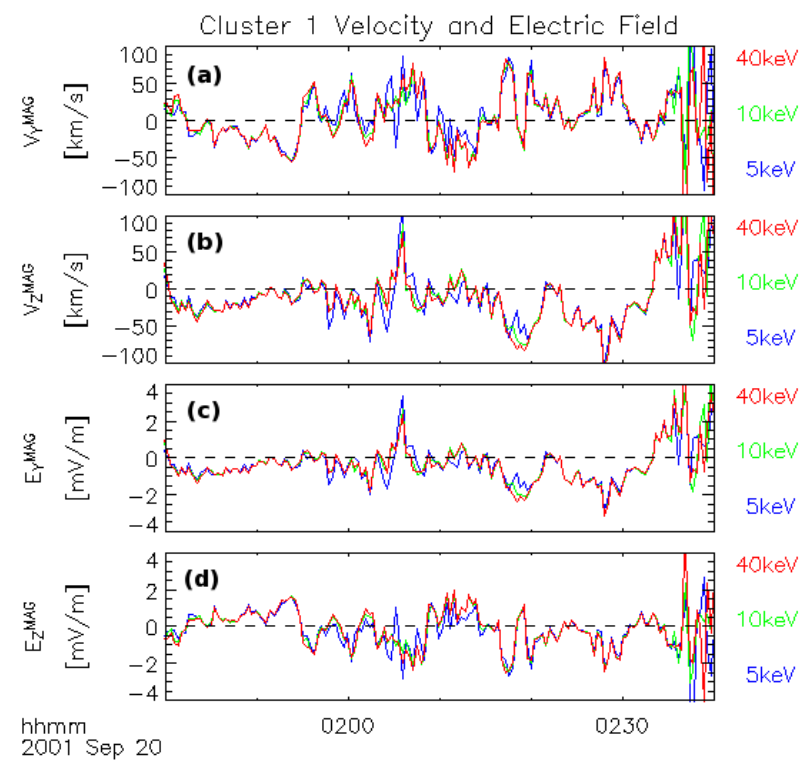

Fig. 9. The $Y_{M A G}$ and $Z_{M A G}$ components of $\boldsymbol{V}(\mathbf{a}, \mathbf{b})$ and $\boldsymbol{E}$ $(\mathbf{c}, \mathbf{d})$, obtained by integration of the 3 -D CODIF $\mathrm{H}^{+}$distributions from $\mathrm{SC} 1$ over three energy ranges, from $40 \mathrm{eV}$ up to $5 \mathrm{keV}$ (blue), $10 \mathrm{keV}$ (green), and $40 \mathrm{keV}$ (red). As for SC1, the results for SC3 and SC4 (not shown) do not exhibit a significant dependence on the upper limit of the energy range.

field line between the reconnection site and Cluster location. In addition, the proton distributions are not very collimated around the magnetic field line, suggesting that the reconnection site is located quite far down the tail - a feature consistent with a substorm growth phase.

The velocity in Fig. 7 was obtained by integrating the $\mathrm{H}+$ distributions measured by CODIF between $W_{\text {min }}=40 \mathrm{eV}$ and $W_{\max }=10 \mathrm{keV}$, which covers the core of the ion plasma (compare with the spectrogram). In order to minimize the influence of the non- $\boldsymbol{E} \times \boldsymbol{B}$ drifts on the velocity, $W_{\max }$ should be taken as low as possible. However, in the plasma sheet $W_{\max }$ should be at least a few $\mathrm{keV}$, otherwise the counting statistic is too poor. In our case, $W_{\max }=10 \mathrm{keV}$ was picked as a reasonable compromise but, as already mentioned in Sect. 3.1.2, the choice of a different value would not cause essential changes in the results.

This point is emphasized by Fig. 9, where we show the SC1 velocity and electric field, obtained for $W_{\max }$ equal to $5 \mathrm{keV}, 10 \mathrm{keV}$, and $40 \mathrm{keV}$ (the full CODIF range). Since we assume $E_{\|}=0$, only the $Y_{M A G}$ and $Z_{M A G}$ components of $\boldsymbol{V}$ and $\boldsymbol{E}$ are given in Fig. 9. It is clear that the choice of $W_{\max }$ does not have much influence on $\boldsymbol{V}$ and $\boldsymbol{E}$. Similar results (not shown) are obtained for SC3 and SC4.

The reader is cautioned that the very small change in $\boldsymbol{E}$ associated with the change in $W_{\max }$ can make a visible difference in the cumulative sum of $\boldsymbol{E} \cdot \boldsymbol{J}$. Small calibration errors resulting in a bias of a few $\mathrm{km} / \mathrm{s}$ in $\boldsymbol{V}$, can lead to a bias of $\sim 0.1 \mathrm{mV} / \mathrm{m}$ in $\boldsymbol{E}$ and of $\simeq 10^{-13} \mathrm{~W}$ in $\boldsymbol{E} \cdot \boldsymbol{J}$. Depending on

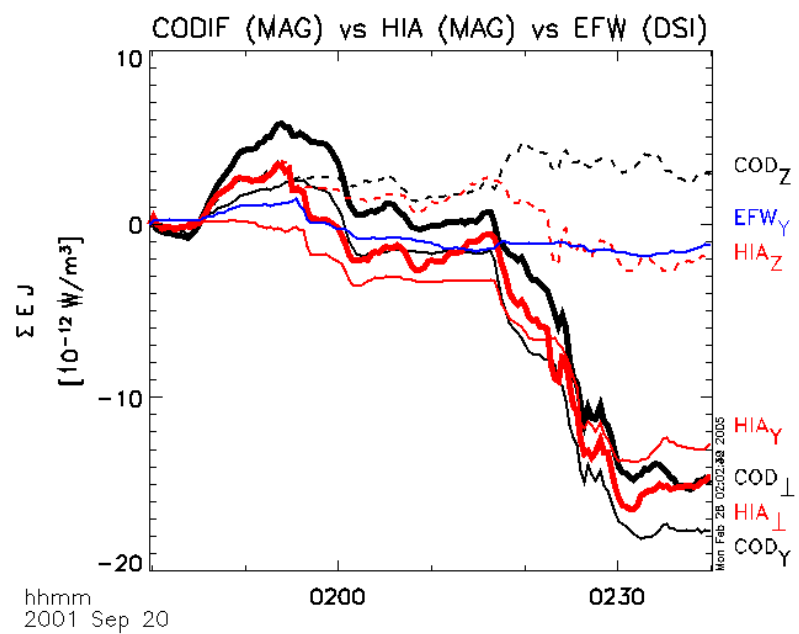

Fig. 10. Cumulative sums of $\boldsymbol{E} \cdot \boldsymbol{J}$, with $\boldsymbol{E}$ estimated from CODIF (black), HIA (red), and EFW (blue) data. In each case the electric field is averaged over the satellites where the respective instrument is operational. For CODIF and HIA the thick solid lines show the total, while the thin solid and dashed lines indicate the contributions from the $Y_{M A G}$ and $Z_{M A G}$ directions. For EFW only the contribution from the $Y_{D S I}$ direction is shown.

the time interval, this implies a bias of a few to a few tens of $\simeq 10^{-12} \mathrm{~W}$ in the cumulative sum. Nevertheless, the key point in this study and in $\mathrm{H} 06$, that is the detection of concentrated regions where $\boldsymbol{E} \cdot \boldsymbol{J}<0$, is not affected by the choice of $W_{\max }$.

Figure 10 expands on the cumulative sum of the power density, by showing CODIF, HIA, and EFW data, including the contributions from $Y_{M A G}$ and $Z_{M A G}$ directions for CODIF and HIA. For EFW we only show the $Y_{D S I}$ contribution because, as discussed in Sect. 3.1.1, the close vicinity of the magnetic field vector to the spacecraft spin plane prevents the complete determination of the electric field.

We chose to present the cumulative sum of the power density, instead of the power density itself, because the trend is easier visible in the cumulative sum. One can compare this procedure with that used for lower altitude satellites, in polar orbits, when the electric field is integrated along the satellite path, in order to obtain a better view on its large-scale trend and on the convection motion. As in this case, one needs reasonably stationary conditions to obtain significant results. Given the quiet magnetospheric conditions (Sect. 4), such an assumption is probably acceptable for the time intervals of the order of $10 \mathrm{~min}$, discussed here and in H06.

The most remarkable feature in Fig. 10 is the good agreement between CODIF and HIA. The thick black and red curves closely follow each other and both of them exhibit a steep negative variation between 02:17 and 02:30. A smaller negative jump in the cumulative sum is seen around 02:00 UT. On the other hand, Fig. 10 shows no evidence of $\boldsymbol{E} \cdot \boldsymbol{J}<0$ in the (blue) EFW data. This feature can be traced 


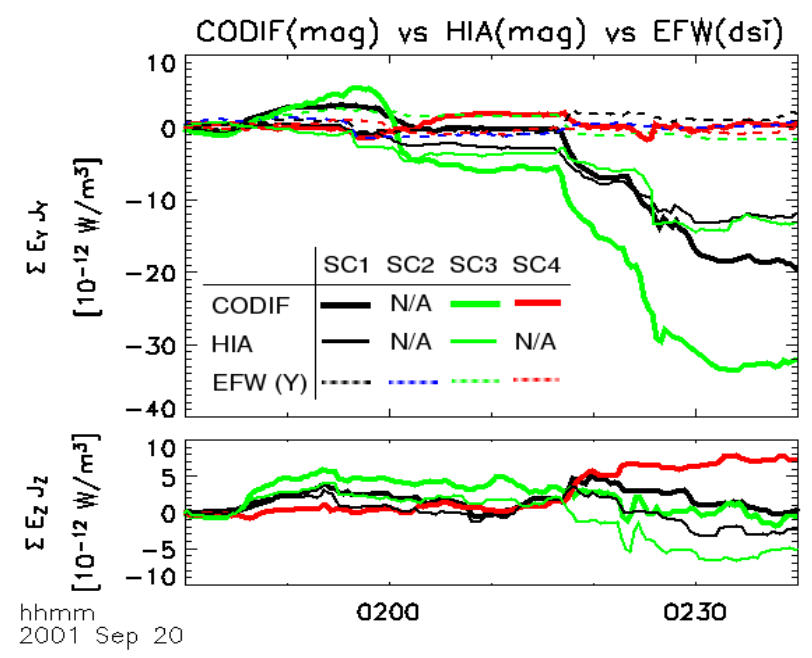

Fig. 11. Cumulative sum of $\boldsymbol{E} \cdot \boldsymbol{J}$ by using the electric field from all available instruments on each spacecraft. The contributions from the $Y$ direction are shown in the top panel and those from the $Z$ direction in the bottom panel. The MAG reference system is used for CODIF and HIA data, DSI for EFW data (only $Y$ direction). The table in the top panel summarizes the colors and linestyles used for each instrument and spacecraft. One notes that the jump in the cumulative sum, indicative for the crossing of a CGR, is mainly exhibited by the $Y$ CODIF and HIA traces on $\mathrm{SC} 1$ and SC3. For the discussion of this feature, see the text.

back to the timing of the conjunction and to the ASPOC instrument being switched off on some of the satellites. A detailed discussion on the validation of the generator signatures is given in next section.

The negative variation indicated by CODIF and HIA in the cumulative sum of $\boldsymbol{E} \cdot \boldsymbol{J}$ between 02:17 UT and 02:30 UT is $\sim 15 \cdot 10^{-12} \mathrm{~W} / \mathrm{m}^{3}$. This implies an average power density $\rho_{W} \equiv \boldsymbol{E} \cdot \boldsymbol{J} \simeq-5 \cdot 10^{-13} \mathrm{~W} / \mathrm{m}^{3}$, consistent with the order of magnitude estimate from Sect. 2.1.

In addition, most of the generator signature is contributed by the $Y$ direction. As discussed below, this is again in good agreement with the expectations. The pressure gradients near the southern plasma sheet boundary are oriented, on average, in the $Z$ direction, which leads to an average $Y$ orientation of the induced diamagnetic currents.

\section{Discussion}

The data presented above support the interpretation that the generator signatures visible in the Cluster data are reliable, and related to the auroral activity detected by FAST. Even if the exact value of the power density may be in error, there is reasonable experimental evidence that Cluster crosses indeed a concentrated generator region (CGR), and that at least part of the generated electromagnetic energy is fed to the auroral ionosphere. In order to substantiate these findings, we concentrate below on: a) additional features which contribute to the validation of the $\boldsymbol{E} \cdot \boldsymbol{J}<0$ signatures; b) the generator mechanism and configuration.

\subsection{Validation of the generator signatures}

We shall start by investigating the discrepancy exhibited by Fig. 10, between CODIF and HIA, on the one hand, and EFW, on the other hand. In order to understand its origin, in Fig. 11 we present the cumulative sum of the power density, computed by using $\boldsymbol{J}$ as derived with the Curlometer and $\boldsymbol{E}$ as measured on each satellite by the available instruments. The contributions from the $Y$ and $Z$ directions are plotted in separate panels.

The combined use of $\boldsymbol{J}$, which is representative for the whole tetrahedron, and single satellite $\boldsymbol{E}$, can be motivated by operational and physical reasons. The average electric field used above is $\left\langle\boldsymbol{E}^{I N S}\right\rangle=\sum_{S C} \boldsymbol{E}_{S C}^{I N S} / N^{I N S}$, where $I N S$ is the instrument, the angular brackets denote the average over spacecraft with available $I N S$ data, and $N^{I N S}$ is the number of such spacecraft. Therefore, $\left\langle\boldsymbol{E}^{I N S}\right\rangle \cdot \boldsymbol{J}$ can be seen as a sum of contributions coming from each spacecraft, which ideally should not depend on the instrument. The use of the single satellite electric field would be physically meaningful if the current does not vary too much over the tetrahedron. The apparent consistency of the results to be obtained below suggests that this might indeed be the case.

The generator signature is visible on $\mathrm{SC} 1$ and $\mathrm{SC} 3$, in the CODIF and HIA data. As in Fig. 10, the negative jump in the cumulative sum of the power density shows up only in the $Y$ contributions. No generator signature is seen on $\mathrm{SC} 2$ (where we only have EFW data) and SC4.

To clarify why EFW does not see the generator, we start by noting that the ASPOC instrument was switched off on $\mathrm{SC} 1$ and SC2. Therefore, the EFW electric field is not very reliable on these satellites (we note that a similar analysis performed for the events presented in $\mathrm{H06}$ leads to the same result: no generator signature is seen on $\mathrm{SC} 1$ and $\mathrm{SC} 2$ when using EFW data). On the other hand, on SC1 there is good agreement between CODIF and HIA in showing a generator, which is supportive for this signature being real. On SC2 there is no data from CODIF or HIA and, with just one operational instrument, we do not have enough direct evidence for or against the generator. However, considerations related to the conjunction timing, given below, suggest that one would rather not expect a generator signature on $\mathrm{SC} 2$.

On SC3 it is more difficult to decide. Here CODIF and HIA show a generator encounter, while EFW does not, although ASPOC is on. Even if in this case the agreement between CODIF and HIA is no longer as good as on SC1, both instruments agree on crossing a CGR. According to the criterion introduced at the beginning of Sect. 3.1, we choose to validate the generator signature on SC3. When the instruments disagree the uncertainty is higher and one cannot exclude that EFW is correct. However, the detailed 
examination of the data to be performed below supports the interpretation that $\mathrm{SC} 3$ crosses a generator region.

On SC4 ASPOC is on and neither EFW nor CODIF show the negative jump in the cumulative sum of the power density, from which we conclude SC4 does not meet the CGR.

The fact that $\mathrm{SC} 1$ and $\mathrm{SC} 3$ encounter a CGR, while SC4 (and probably SC2) does not, can be explained by both temporal and spatial variability. Figure 12 zooms in on the tail projection of FAST, and the traces of the four Cluster satellites, together with their mass center, in the $\left(Y_{G S M}, Z_{G S M}\right)$ plane. Not visible in Fig. 12 is the separation along the $X$ axis: $\mathrm{SC} 1$ is the leading satellite, followed by $\mathrm{SC} 2, \mathrm{SC} 3$, and SC4 at $500 \mathrm{~km}, 1400 \mathrm{~km}$, and $1900 \mathrm{~km}$, respectively. In order to provide an approximate measure for the uncertainties associated with the mapping, we show the tail footprints of FAST for both $R=18 R_{E}$ and $R=20 R_{E}$ (the average geocentric distance of Cluster at the conjunction time is $18.7 R_{E}$ ).

As proved by the magnetic field and electron data (Sect. 5.1), the auroral form overpassed by FAST between 02:36 UT and 02:37 UT has the characteristics of a faint quiet arc, elongated in azimuthal direction. The tail projection of this auroral form should be accordingly elongated in the $Y_{G S M}$ direction. Consequently, we believe that the energy flux measured by FAST around 02:36 UT is related to the CGR crossed by SC1 and SC3 between 02:17 UT and 02:30 UT. By this we do not imply that the CGR is necessarily elongated in $Y_{G S M}$ direction. As discussed in the next section, it is possible that the arc is powered by a collection of CGRs, at different locations on different field lines.

If the data measured by SC1 and SC3 between 02:17 UT and 02:30 UT are indeed related to the data measured by FAST around 02:36 UT, the lifetime of the CGR should be 20 min or more - consistent with the hypothesis of a quiet arc. Such a lifetime is long enough to allow for SC4, whose projection in the $\left(Y_{G S M}, Z_{G S M}\right)$ plane is about $10 \mathrm{~min}$ behind the projection of SC1, to reach the CGR field lines. The fact that no generator signature is detected on SC4 sets a constraint on the CGR extension along the field line, $L_{G_{X}}$. We note that in $20 \mathrm{~min}$ SC4 (which is $1900 \mathrm{~km}$ behind SC1 and $500 \mathrm{~km}$ behind SC3) travels $\sim 800 \mathrm{~km}$ along the field line (at a speed of $\sim 0.65 \mathrm{~km} / \mathrm{s}$ ) towards the CGR. This enforces the constraint on the CGR extension in the $X$ direction and, in addition, suggests that some temporal variability is also needed.

An estimate for $L_{G_{X}}$ can be obtained along the same line used in Sect. 2.1 to evaluate the order of magnitude for the power density in the generator region. With a measured power density, $\rho_{W} \simeq 5 \cdot 10^{-13} \mathrm{~W} / \mathrm{m}^{3}$, a measured ionospheric energy flux, $\Phi_{W} \simeq 10^{-3} \mathrm{~W} / \mathrm{m}^{2}$, and a surface mapping factor $R \simeq 1700$, we obtain $L_{G_{X}} \simeq \Phi_{W} /\left(R \cdot \rho_{W}\right) \simeq 1000 \mathrm{~km}$. This value is comparable to the separation between the Cluster satellites in the $X$ direction, and is compatible with our constraint.

It should be pointed out, however, that $1000 \mathrm{~km}$ is probably a lower limit for $L_{G_{X}}$. Not all the energy converted from

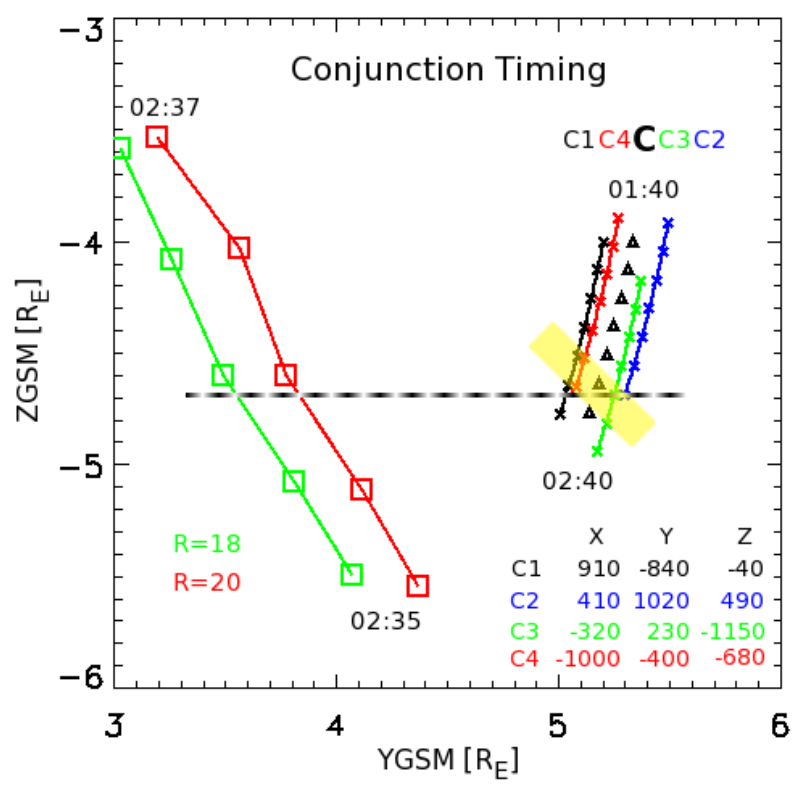

Fig. 12. Tail footprint of FAST, in the $\left(Y_{G S M}, Z_{G S M}\right)$ plane, together with the projections of the four Cluster spacecraft and of the tetrahedron mass center. The FAST footprint, found by using the T96 model, is shown for both $18 R_{E}$ and $20 R_{E}$, in order to provide an approximate measure for the mapping uncertainties. The FAST footprint is shown with squares, every $30 \mathrm{~s}$, while the Cluster traces are marked with " $\mathrm{x}$ " every $10 \mathrm{~min}$. The mass center of the Cluster tetrahedron is indicated with triangles. The spacecraft coordinates relative to the tetrahedron mass center (in $\mathrm{km}$ ) are given in the lower right corner. The conjunction with $\mathrm{SC} 1$ is indicated with dashed horizontal line. The shaded area shows roughly the CGR projection near Cluster, as inferred from the generator signatures on $\mathrm{SC} 1$ and SC3.

mechanical to electromagnetic is carried by Alfvén waves to the ionosphere (see also Sect. 6.2), and not all the energy carried to the ionosphere is deposited there, because of the Alfvén waves' reflection. $L_{G_{X}}$ is probably larger than $1000 \mathrm{~km}$, but we believe not by orders of magnitude larger. Besides the constraint related to the absence of the generator signature in the SC4 data, a rather limited $L_{G_{X}}$ would be consistent with the substantial $\nabla \cdot \boldsymbol{B} /|\nabla \times \boldsymbol{B}|$ ratio during the CGR encounter (panel (d) of Fig. 7). If the structure crossed by Cluster is comparable to the tetrahedron characteristic size, one can expect nonlinear variations in the magnetic field, which can lead to errors when applying the Curlometer. Nevertheless, we will show in the next section that the current provided by the Curlometer agrees with the expectations near the plasma sheet boundary.

Before discussing in more detail the generator mechanism, it is worth remembering that the $L_{G_{X}}$ estimate used in Sect. 2.1 was 1-2 orders of magnitude higher than the value suggested above. However, the estimate from Sect. 2.1 refers to the large-scale auroral current circuit, while our CGR appears to be rather a small-scale feature. As discussed below, 

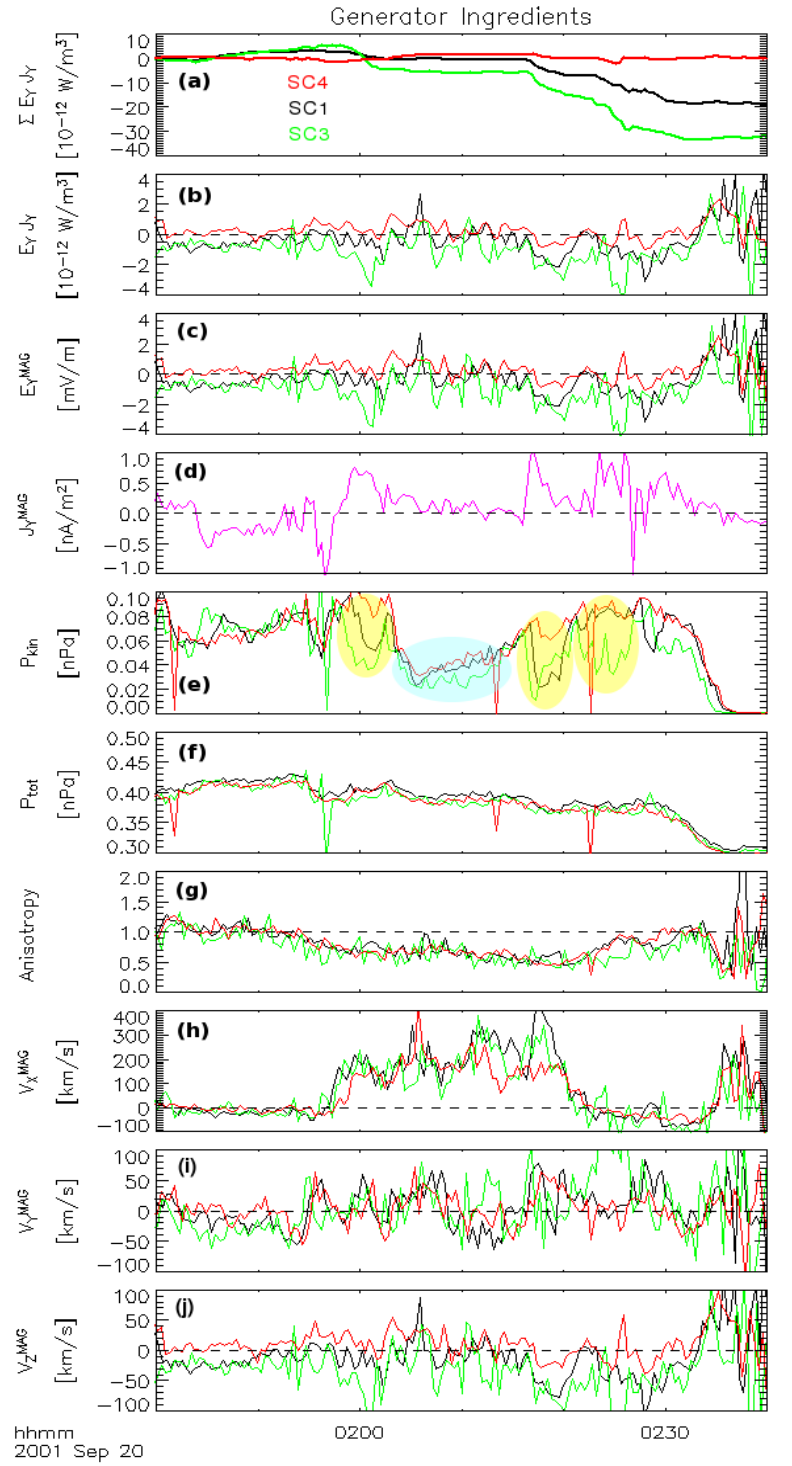

Fig. 13. Cumulative sum of $E_{Y} J_{Y}$ (a), $E_{Y} J_{Y}$ (b), $E_{Y}$ (c), $J_{Y}$ (d), kinetic (e) and total (f) pressure, anisotropy (g), and the velocity components $(\mathbf{h}-\mathbf{j})$. All the quantities are shown for SC1 (black), SC3 (green), and SC4 (red), except for the current, which is derived by the Curlometer and representative for the whole tetrahedron. The plasma moments are derived from CODIF data. The yellow shading in panel (e) emphasizes the intervals with substantial $P_{k i n}$ gradients, while the cyan shading indicates the time in between, when SC1, SC3, and SC4 measure about the same $P_{k i n}$.

it is possible that several CGRs near the plasma sheet/lobe interface play a significant role in powering the aurora close to the PCB, at least during quiet times.

\subsection{Generator mechanism and configuration}

The detection of a generator signature in the plasma sheet, close to its boundary, is not surprising. This is a proper location to find diamagnetic electric currents, because of the gradients in the pressure, $\boldsymbol{j}=-\nabla \cdot \mathbf{P} \times \boldsymbol{B} / B^{2}$ (with $\mathbf{P}$ the pressure tensor). It is also a proper location to find electric fields, induced by the plasma motion and related to the oscillations one can always expect at a plasma interface. Currents and electric fields are necessary but not sufficient ingredients for a generator. In order to make a generator, one needs, in addition, conversion of mechanical energy into electromagnetic energy (mostly magnetic in our case), that is the proper relation between the current and the electric field, $\boldsymbol{E} \cdot \boldsymbol{J}<0$. Still, this generator is not necessarily connected to the aurora. The energy may be just converted locally back and forth, or circulated in the magnetosphere.

In this section we try to accomplish two goals: first, we investigate in more detail how consistent are the ingredients with our CGR signature. Second, we discuss whether this CGR is indeed supplying energy to be dissipated in the auroral ionosphere. The first goal is easier to attain, because it requires only analysis of the quantities measured locally. The second goal raises more difficult problems, because we do not have any possibility to really follow what happens to the generated electromagnetic energy. The FAST measurements favor the interpretation that at least part of this energy finally dissipates in the ionosphere, but do not exclude other alternatives.

The generator ingredients are presented in Fig. 13. We chose to show only the cumulative sum of $E_{Y} J_{Y}$ (a), $E_{Y} J_{Y}$ (b), $E_{Y}$ (c), and $J_{Y}$ (d), because the $Y$ direction makes the dominant contribution to the generator. In the other panels we show the kinetic (thermal) pressure, $P_{\text {kin }}(\mathrm{e})$, the total pressure, kinetic plus magnetic, $P_{t o t}=P_{k i n}+P_{m a g}(\mathrm{f})$, the anisotropy $(\mathrm{g})$, and the three components of the velocity (hj). All of these quantities are derived by using CODIF data from $\mathrm{SC} 1, \mathrm{SC} 3$, and $\mathrm{SC} 4$, in the MAG reference system.

A prominent feature in the pressure panels is the substantial variation in $P_{k i n}$, different from satellite to satellite (yellow shading), at the same time with rather small fluctuations in $P_{t o t}$, which is almost the same on the three satellites. At 01:58, coincident with the entry in the earthward flow region (EFR), exhibited by $V_{x}(\mathrm{~h})$, we see an abrupt variation in $P_{k i n}$ on SC3 and, a bit later, on SC1 (e). After a short recovery at $02: 03, P_{k i n}$ reaches a minimum of $\sim 0.03 \mathrm{nPa}$ around 02:05 and then it returns to $\sim 0.07 \mathrm{nPa}$ on all three satellites (cyan shading). Before 02:20 $P_{k i n}$ reaches another minimum, mainly on SC1 and SC3, during the final part of the EFR. Another short recovery is followed by a new minimum on SC3, during the second half of the CGR. Finally, around 02:33, $P_{k i n}$ starts the last steep decline to its lobe value, approximately at the same time on all three satellites.

Even at the times when $P_{k i n}$ shows large differences from satellite to satellite, $P_{t o t}$ exhibits little dependence on the satellite. This indicates that the $P_{\text {kin }}$ variation depends on the satellite distance to the plasma sheet boundary, consistent with the fact that most of the time $P_{\text {kin }}$ takes its smallest value on SC3 - which has the most negative $Z$ coordinate, 
as seen in Fig. 12. However, the distance to the plasma sheet boundary does not depend only on $Z$, but also on $X$ and $Y$ : between 02:05 and 02:15 $P_{k i n}$ is about the same on SC1, SC3, and SC4, while between 02:17 and 02:20 about the same minimum in $P_{k i n}$ is seen on both SC1 and SC3. This suggests a complicated, 3-D wavy structure of the boundary, consistent with the discussion of the conjunction timing in Sect. 6.1. As already pointed out there, the characteristic size of this structure appears to be of the same order with the distance between the satellites, that is up to a few $1000 \mathrm{~km}$.

By comparing the current in panel (d) with the pressures in panel (e), it becomes clear that the peaks in the current are collocated with the large differences in $P_{k i n}$ between the satellites, consistent with the diamagnetic character of the current. This qualitative agreement can be substantiated by a rough quantitative estimate. For example, at the center of the CGR, around 02:24, the difference between $P_{k i n}$ on SC1 and SC4, and $P_{k i n}$ on SC3, is $\Delta P_{k i n} \simeq 0.05 \mathrm{nPa}$, over a distance $L \simeq 1000 \mathrm{~km}$. With a magnetic field $B \simeq 30 \mathrm{nT}$, one obtains a diamagnetic current $J_{d} \simeq \Delta P_{k i n} / B L \simeq 1.7 \mathrm{nA} / \mathrm{m}^{2}$, which fits pretty well with $J_{Y} \simeq 1 \mathrm{nA} / \mathrm{m}^{2}$ from panel (d), obtained via the Curlometer method from totally different data.

For a more precise estimate of the current one would have to take into account the contribution related to the pressure anisotropy $(\mathrm{g})$ and the curvature of the magnetic field, $J_{c}=\left(p_{\|}-p_{\perp}\right) /\left(B^{2} R_{c}\right) \boldsymbol{B} \times \boldsymbol{n}$ (e.g. Ferraro and Plumpton, 1966, p. 219-220). $R_{c}$ is the curvature radius of the field line which can be computed from the multi-point magnetic field data, and $\boldsymbol{n}$ is the unit vector along the principal normal. In the vicinity of a wavy boundary which varies along distances of up to a few $1000 \mathrm{~km}$, one can expect that $R_{c}$ is also of this order, and $J_{c}$ can make a non-negligible contribution to the current. For example, with $p_{\|} \simeq 0.1 \mathrm{nPa}$, $0.05 \mathrm{nPa} \lesssim P_{\perp} \lesssim 0.1 \mathrm{nPa}$ (which results in an anisotropy between 0.5 and 1 , as in panel (g) of Fig. 13), $B \simeq 30 \mathrm{nT}$, and $R_{c} \simeq 5000 \mathrm{~km}$, one obtains $J_{c}$ between 0 and $0.3 \mathrm{nA} / \mathrm{m}^{2}$. Depending on the orientation of $\boldsymbol{n}, J_{c}$ can add to or subtract from $J_{d}$.

Further insight into the generator mechanism is obtained by inspecting the plasma velocity. One can see that the negative $E_{Y}$ electric field on SC1 and SC3 during the CGR encounter is related to the negative $V_{Z}$ velocity. On $\mathrm{SC} 4$, where we see no generator signature, $V_{Z}$ stays, on average, around zero. It is noteworthy that $V_{Z}$ is negative on $\mathrm{SC} 1$ and $\mathrm{SC}$, shortly before the two satellites leave the plasma sheet, while $V_{Y}$ is, on average, positive. The plasma velocity in the close vicinity of the boundary is about equal to the velocity of the boundary. In order to have the satellites leaving the plasma sheet, the plasma sheet boundary near the spacecraft should be tilted with respect to the $Y$ direction, consistent with the orientation of the CGR projection in Fig. 12. A similar velocity (and pressure) pattern is seen around 02:00 UT, when $\mathrm{SC} 1$ and SC3 encounter the smaller generator region.

By looking at the time series data in Fig. 13, the small CGR around 02:00 UT and the main CGR starting at 02:17 appear to bound the EFR. This raises the question as to whether there is any causal relationship between the two CGRs and the EFR. We note that no generator signature $(a, b)$ is seen within the central part of the EFR, between 02:01 UT and 02:17 UT. During this time there is about the same $P_{k i n}$ on SC1, SC3, and SC4, consistent with the vanishing current and with the presumption that all the satellites (including SC2) were probing pretty much the same layer, within about the same distance from the plasma sheet boundary. A different situation is presented in H06, where a CGR, found to be collocated with an EFR, is interpreted in terms of plasma sheet expansion traveling over the spacecraft. In that case, however, both the CGR and the EFR are quite narrow ( $\sim 4 \mathrm{~min}$ ) and collocated. In addition, the data are measured during a substorm recovery phase, when the plasma sheet is indeed expected to expand.

In our case, the data is measured during the growth phase of a small substorm, the EFR is wide, and for most of it there is no generator signature. Before drawing a more definitive conclusion, at least a few other similar events need to be identified and explored, but we believe that our two CGRs are not causally related to the EFR. Rather, the wavy motion of the plasma sheet boundary is sometimes bringing CGRs at the right position to be measured by Cluster.

One problem implied by such a scenario is that near a wavy plasma sheet surface it is easier to imagine energy that is exchanged locally, than energy transported to the ionosphere. However, since the Alfvén waves are known to couple mechanical and electromagnetic oscillations, it is conceivable that at some points the right conditions are realized, so that traveling surface waves (launched, for example, at the magnetopause) are able to excite Alfvén waves, which propagate and carry the energy to the ionosphere.

A hint in this direction is provided by a closer examination of the Poynting theorem:

$\nabla \cdot \boldsymbol{S}=-\frac{\partial W_{m a g}}{\partial t}-\boldsymbol{E} \cdot \boldsymbol{J}$

where $\boldsymbol{S}=\boldsymbol{E} \times \boldsymbol{B} / \mu_{0}$ is the flux of electromagnetic energy (the Poynting flux) and $W_{\text {mag }}$ is the electromagnetic energy density, dominated in the plasma by the magnetic term. $W_{\text {mag }}$ is identical to the magnetic pressure:

$W_{m a g} \equiv P_{m a g}=\frac{B^{2}}{2 \mu_{0}}$.

When $\nabla \cdot S>0$, electromagnetic flux is generated within the volume element and can be carried away, for example, by Alfvén waves. If $\boldsymbol{E} \cdot \boldsymbol{J}<0$, a sufficient condition for $\nabla \cdot \boldsymbol{S}>0$ is to have $\partial P_{\text {mag }} / \partial t<0$. When $\partial P_{\text {mag }} / \partial t>0$, the two terms on the right side of Eq. (1) compete and a more careful quantitative evaluation is needed.

The data we have allow for a rough evaluation of $\partial P_{\text {mag }} / \partial t$. Since the Cluster velocity is small compared to the plasma velocity, we can approximate that Cluster is at rest in GSE and compute $\partial P_{m a g} / \partial t$ from the measured data. 
As the total pressure, $P_{t o t}=P_{k i n}+P_{\text {mag }}$, is pretty much constant, $\partial P_{m a g} / \partial t \simeq-\partial P_{k i n} / \partial t$. Panels (a) and (e) of Fig. 13 show that when the generator signature is seen there are times with $\partial P_{k i n} / \partial t>0$ on $\mathrm{SC} 1$ and/or SC3.

Because $\boldsymbol{E} \cdot \boldsymbol{J}$ is computed by using the average current over the tetrahedron, while $\partial P_{\text {mag }} / \partial t$ is estimated for each spacecraft, we cannot exclude the possibility that the regions in space where $\boldsymbol{E} \cdot \boldsymbol{J}<0$ are different from those with $\partial P_{\text {mag }} / \partial t<0$. However, the discussion above suggests that there is electromagnetic energy flux originating from the CGRs. Additional evidence in this direction and more discussion on the Poynting flux and energy conversion issues are given in $\mathrm{H} 06$.

The emission of electromagnetic energy flux from an individual CGR does not mean yet that this energy is going to be fed into the auroral ionosphere. However, a collection of CGRs embedded in a dynamic, wavy structure near the plasma sheet/lobe interface, could dump a net energy flux into the ionosphere. It is beyond the scope of this paper to model this structure and its coupling to Alfvén waves. We just note that within such a scenario it is possible to match a dynamic generator region, consisting of many CGRs in the vicinity of the plasma sheet/lobe interface, to a quiet, elongated arc, near the PCB. While the locations of the CGRs providing the energy to be carried by Alfvén waves may vary with time on each magnetic field line, the energy supplied to the ionosphere and therefore the net auroral effect could show little time dependence.

Such a mechanism might contribute a significant fraction of the auroral energy dissipated at quiet times near the PCB. When the magnetosphere is more active, rapid changes in the plasma flow configuration in the vicinity of the reconnection site in the tail are communicated to the ionosphere by Alfvén waves. They propagate along magnetic field lines near the plasma sheet boundary and probably dominate the CGRs related Alfvén activity. When the magnetosphere is quiet, however, the tail reconnection is expected to proceed more or less stationary, and the intensity of the Alfvén waves launched from the reconnection site is reduced. On such occasions the CGRs may represent the main energy source for the auroral activity close to the PCB.

\section{$7 \quad$ Summary and prospects}

In this paper we presented in-situ experimental evidence for the crossing of concentrated generator regions (CGRs), based on conjugate Cluster and FAST data. It is the first time that experimental data are used to compute the power density and identify generator domains, where $\boldsymbol{E} \cdot \boldsymbol{J}<0$. Two capabilities available on Cluster were essential to attain this goal: the possibility to fully resolve the current vector, and the redundancy in the electric field information. The method developed here allowed for reasonable estimates of the power density, even if the measured data were close to the instrumental detection limits.

The CGRs were encountered by Cluster at $18 R_{E}$, in the vicinity of the plasma sheet boundary. For the main structure detected, the negative power density was equal, on average, to $-0.5 \cdot 10^{-12} \mathrm{~W} / \mathrm{m}^{3}$ and was seen for more than $10 \mathrm{~min}$. This signature was found to be consistent with conjugate auroral activity measured by FAST at $0.6 R_{E}$, near the polar cap boundary (PCB), and with the geophysical conditions. Although one cannot follow the energy propagation along the magnetic field line, the conjunction between CGRs at high altitude and energetic electron precipitation at low altitude suggests that at least part of the generated energy is indeed used to power the aurora.

The location of Cluster and FAST close to the boundary between the plasma sheet and the lobe was a key feature in our study. On the one hand, it provided us with the operational means to cross-check important pieces of information - like the magnetic field line mapping by using particle data, or the current density obtained with the Curlometer by using the pressure gradient. On the other hand, the vicinity of the plasma sheet/lobe interface provided the ingredients needed for the generator: electric fields coupled to the plasma motion and related to the oscillations of the plasma sheet boundary, as well as electric currents induced by the pressure gradients.

The CGRs proved to have a complicated 3-D structure, extending up to a few $1000 \mathrm{~km}$ along the magnetic field line. Consistent with the apparently wavy character of this structure, and with the conjugate energy flux into the ionosphere, we suggested the possibility that the CGRs couple to Alfvén waves, able to carry away their energy and dump it into the auroral oval, near the PCB. Several CGRs, distributed at various locations along different magnetic field lines, could represent the main energy source for auroral activity in the vicinity of the PCB, during quiet magnetospheric intervals.

We consider the results obtained as promising and feel encouraged to continue the experimental search for auroral generator regions. By using the same method as here we identified a few other CGRs, which are presented in H06. These CGRs also exhibit complicated 3-D structures which require a closer examination. Another topic that requires future work is the coupling between the surface waves propagating at the plasma sheet boundary and the Alfvén waves. However, before undertaking steps in these rather theoretical and modeling directions, better event statistic and more work on measured data are needed.

Acknowledgements. We are pleased to thank A. Blăgău, M. Bouhram, T. Karlsson, Y. Khotyaintsev, G. Marklund, G. Paschmann, K. Rönnmark, and H. Vaith for fruitful discussions. O. Marghitu acknowledges the kind hospitality of Max-Planck-Institut für extraterrestrische Physik, Garching, and the support by the German Bundesministerium für Bildung und Forschung and the Zentrum für Luft- und Raumfahrt under contract 50 OC 0102 . The work in Romania was funded through the program AEROSPAŢIAL, contracts 72/2003 PROSPERO 
and 118/2004 DIAFAN. We also acknowledge the World Data Center for Geomagnetism, Kyoto, for the $A E$ and $D_{s t}$ indices, the GeoForschungsZentrum (GFZ), Potsdam, for the $K_{p}$ index, the ACE magnetic field instrument (MFI), and plasma instrument (SWEPAM) teams, for the solar wind parameters, as well as the Satellite Situation Center (SSCWeb), for the conjunction query tool.

Topical Editor T. Pulkkinen thanks J. Vogt and another referee for their help in evaluating this paper.

\section{References}

Alfvén, H. and Fälthammar, C.-G.: Cosmical Electrodynamics, The International Series of Monographs on Physics, Clarendon Press, Oxford, 1963.

Balogh, A., Carr, C. M., Acuña, M. H., et al.: The Cluster Magnetic Field Investigation: Overview of in-flight performance and initial results, Ann. Geophys., 19, 1207-1217, 2001.

Birn, J. and Hesse, M.: Details of current disruption and diversion in simulations of magnetotail dynamics, J. Geophys. Res., 101, $15345-15358,1996$.

Birn, J., Hesse, M., and Schindler, K.: MHD simulations of magnetotail dynamics, J. Geophys. Res., 101, 12 939-12 954, 1996.

Brekke, A., Nozawa, S., and Sparr, T.: Studies of the $E$ region neutral wind in the quiet auroral ionosphere, J. Geophys. Res., 99, 8801-8825, 1994.

Carlson, C., McFadden, J., Turin, P., Curtis, D., and Magoncelli, A.: The electron and ion plasma experiment for FAST, Space Sci. Rev., 98, 33-66, 2001.

Dunlop, M., Balogh, A., Glassmeier, K.-H., and Robert, P.: Four-point Cluster application of magnetic field analysis tools: The Curlometer, J. Geophys. Res., 107, 1384, doi:10.1029/2001JA005088, 2002.

Elphic, R., Means, J., Snare, R., Strangeway, R., and Kepko, L.: Magnetic field instruments for the Fast Auroral Snapshot explorer, Space Sci. Rev., 98, 151-168, 2001.

Eriksson, A., André, M., Klecker, B., et al.: Electric field measurements on Cluster: Comparing the double-probe and electron drift techniques, Ann. Geophys., 24, 275-289, 2006.

Escoubet, C. P., Fehringer, M., and Goldstein, M.: The Cluster mission, Ann. Geophys., 19, 1197-1200, 2001.

Ferraro, V. and Plumpton, C.: An Introduction to Magneto-Fluid Mechanics, Clarendon Press, Oxford, 1966.

Gustafsson, G., André, M., Carozzi, T., et al.: First results of electric field and density observations by Cluster EFW based on initial months of operation, Ann. Geophys., 19, 1219-1240, 2001.

Hamrin, M., Marghitu, O., Rönnmark, K., et al. : Observations of concentrated generator regions in the nightside magnetosphere by Cluster/Fast conjunctions, Ann. Geophys., 24, 637649, 2006.

Holzworth, R. and Meng, C.: Mathematical representation of the auroral oval, Geophys. Res. Lett., 2, 377-380, 1975.
Klumpar, D., Möbius, E., Kistler, L., et al.: Time-of-flight Energy, Angle, Mass Spectrograph (TEAMS) experiment for FAST, Space Sci. Rev., 98, 197-219, 2001.

Lysak, R.: Auroral electrodynamics with current and voltage generators, J. Geophys. Res., 90, 4178-4190, 1985.

Nozawa, S. and Brekke, A.: Studies of the $E$ region neutral wind in the disturbed auroral ionosphere, J. Geophys. Res., 100, $14717-$ 14734, 1995.

Paschmann, G. and Daly, P. (Eds.).: Analysis Methods for Multi-Spacecraft Data, vol. SR-001 of ISSI Scientific Report, ISSI/ESA, Bern, Switzerland, 1998.

Paschmann, G., Quinn, J., Torbert, R., et al.: The Electron Drift Instrument on Cluster: Overview of first results, Ann. Geophys., 19, 1273-1288, 2001.

Paschmann, G., Haaland, S., and Treumann, R. (Eds.): Auroral plasma physics, no. 15 in: Space Science series of ISSI, Kluwer, Dordrecht, 2003.

Pfaff, R., Carlson, C., Watzin, J., Everett, D., and Gruner, T.: An overview of the Fast Auroral SnapshoT (FAST) satellite, Space Sci. Rev., 98, 1-32, 2001.

Rème, H., Aoustin, C., Bosqued, J., et al.: First multispacecraft ion measurements in and near the Earth's magnetosphere with the identical Cluster ion spectrometry (CIS) experiment, Ann. Geophys., 19, 1303-1354, 2001.

Robert, P., Dunlop, M., Roux, A., and Chanteur, G.: Accuracy of current density determination, pp. 395-418, vol. SR-001 of Paschmann and Daly (1998), 1998a.

Robert, P., Roux, A., Harvey, C., Dunlop, M., Daly, P., and Glassmeier, K.-H.: Tetrahedron geometric factors, vol. SR-001 of Paschmann and Daly (1998), 323-348, 1998b.

Rostoker, G. and Boström, R.: A mechanism for driving the gross Birkeland current configuration in the auroral oval, J. Geophys. Res., 81, 235-244, 1976.

Torkar, K., Riedler, W., Escoubet, C. P., et al.: Active spacecraft potential control for Cluster - implementation and first results, Ann. Geophys., 19, 1289-1302, 2001.

Tsyganenko, N.: Modeling the Earth's magnetospheric magnetic field confined within a realistic magnetopause, J. Geophys. Res., 100, 5599-5612, 1995.

Tsyganenko, N.: A model of the near magnetosphere with a dawndusk asymmetry, 1. Mathematical structure, J. Geophys. Res., 107, 1179, doi:10.1029/2001JA000219, 2002a.

Tsyganenko, N.: A model of the near magnetosphere with a dawndusk asymmetry 1. Parametrization and fitting to observations, J. Geophys. Res., 107, 1176, doi:10.1029/2001JA000220, 2002b.

Vogt, J., Haerendel, G., and Glassmeier, K.-H.: A model for the reflection of Alfvén waves at the source region of the Birkeland current system: The tau generator, J. Geophys. Res., 104, 269278, 1999.

Wygant, J., Keiling, A., Cattell, C., et al.: Polar spacecraft based comparisons of intense electric fields and Poynting flux near and within the plasma sheet - tail lobe boundary to UVI images: An energy source for the aurora, J. Geophys. Res., 105, $18675-$ $18692,2000$. 